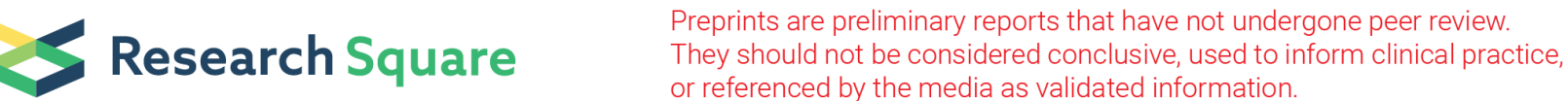

\section{Effects of Dietary Soy Isoflavone and Soy Saponin on Growth Performance, Intestinal Structure, Intestinal Immunity and Gut Microbiota Community on Rice Field Eel (Monopterus Albus)}

\author{
Yajun Hu \\ Hunan Agricultural University \\ Wuying Chu \\ Changsha University \\ Junzhi Zhang \\ Hunan Agricultural University \\ Yi Hu (D huyi740322@163.com ) \\ Hunan Agricultural University https://orcid.org/0000-0002-0185-9250
}

\section{Research}

Keywords: Soy isoflavone, Soy saponin, Growth performance, Intestinal structure, Intestinal immunity, Gut microbiota community, Monopterus albus

Posted Date: December 17th, 2020

DOI: https://doi.org/10.21203/rs.3.rs-129115/v1

License: @ (1) This work is licensed under a Creative Commons Attribution 4.0 International License. Read Full License 


\section{Abstract}

Background: Soy isoflavone and soy saponin are heat-stable glycosides present in soybean meal, which is the major factor restrict soy protein using in aquatic feed. This study is aimed to conduct that dietary soy isoflavone and soy saponin on growth performance, intestinal structure, intestinal immunity and gut microbiota community on rice field eel (Monopterus albus), and revealed its mechanism.

Results: In current study, dietary $1 \mathrm{~g} / \mathrm{kg}$ soy isoflavone, $2 \mathrm{~g} / \mathrm{kg}$ soy saponin and their mixed feed decreased growth of $M$. albus. Dietary $1 \mathrm{~g} / \mathrm{kg}$ soy isoflavone significantly increased serum alkaline phosphatase $(P<0.05)$, dietary $2 \mathrm{~g} / \mathrm{kg}$ soy saponin remarkably declined intestinal immunoglobulin $\mathrm{M}(P<0.05)$. Dietary $1 \mathrm{~g} / \mathrm{kg}$ soy isoflavone and $2 \mathrm{~g} / \mathrm{kg}$ soy saponin mixed feed significantly decreased intestinal complement 3, complement 4 and immunoglobulin $\mathrm{M}$, amounts of intestinal goblet cells per root and shannon of gut microbiota $(P<0.05)$. Dietary $1 \mathrm{~g} / \mathrm{kg}$ soy isoflavone, $2 \mathrm{~g} / \mathrm{kg}$ soy saponin and their mixed feed down regulated intestinal tight junction protein (Interleukin-12, Interleukin-15, Tight junction protein ZO-2-like) and anti-inflammatory (Interleukin-10, Transforming growth factor beta 2) mRNA expression, up regulated pri-inflammatory factors (Interleukin-1 $\beta$, Interleukin-6, Interleukin-8, Interleukin-12 and Interleukin-15) mRNA expression.

Conclusion: Based on the current results, dietary soy isoflavone and soy saponin could damage the intestinal structure and changes gut bacteria community of $M$. albus, and destroyed the intestinal barrier, eventually induced intestinal inflammatory occurring, soy isoflavone and soy saponin mixed feed has more serious effect than they single supplemented.

\section{Introduction}

Fish meal (FM) is a high-quality protein source for aquatic feed because of its balance of amino acids, high fatty acid content and low carbohydrate content. With the continuous development of aquaculture industry in recent years, the demand for FM has increased rapidly. At the same time, due to climate change and with the increase of global fishing, the global fish resources have declined sharply and the prices have soared. Nevertheless, the probability of hunting for wild marine fish is gradually difficult, conventionally used proteins has considered to be no longer sustainable from both economic and ecologic points of reality view ${ }^{[1]}$.

Soybean protein is a kind of high-quality plant protein, it's also the largest production plant protein in the world, and its short growth cycle. Recently, the aquatic feed industry rapidly expanded, soybean protein is widely used to replace FM in aquatic feed ${ }^{[2]}$. However, various anti-nutritional factors like trypsin inhibitors, antivitamins, lectins, soy antigens, isoflavone, saponins, phytic acid,

phytoestrogens and oligosaccharides are major shortcoming of soybean products used in aquatic feeds ${ }^{[3]}$. Lectins, trypsin inhibitors and antivitamins are heat labile factors could be destroyed by high temperature and extruded treatment, and phytates and antigenic compounds in aquatic feeds containing commercially available soybean protein sources did not affect growth performance and immunity of fish ${ }^{[4]}$. The reasons is the presence of some anti-nutritional factors (ANFs) in variable amounts in the raw soybean grain. The ANFs in soybean mainly consist of heat labile and heat stable factors ${ }^{[5]}$. The process of extruding for soybean is acknowledged to be very useful to remove heat labile ANFs, however, there are thermal stability ANFs in extruded soybean meal has been reported that not only has the negative effect on growth performance, but also damage the intestinal function ${ }^{[6,7]}$.

Soy isoflavone are heat-stable glycosides present in soybean meal, also widely known to exert oestrogen like biological effects on animals because of the similarity of their structure to the natural oestrogen structure ${ }^{[8]}$. There has many physiological function about soy isoflavone, especially for immune system regulation ${ }^{[9]}$, include effects of antioxidant, anti-inflammatory on white shrimp (Litopenaeus vannamei) ${ }^{[10]}$, and improve the immune response and against Aeromonas hydrophila challenge on juvenile grass carp (Ctenopharyngodon idella ${ }^{[11]}$. However, soy isoflavone affected growth performance and altered the physiological processes of on Atlantic salmon (Salmo salarL.) ${ }^{[12]}$. Obviously, effect of soy isoflavone aquatic animals is positive or negative? It's hard to say.

Soy saponin is the main heat-stable glycosides present in soybean meal, even extruded soybean meal ${ }^{[13]}$, and it's also a kind of membrane permeabilising activity ${ }^{[14]}$. Soy saponin is considered to have negative effects for feed intake, weight gain, ability uptake of nutrients including minerals and vitamins in intestine, and declining the digestibility of protein ${ }^{[6,7,15]}$. Generally, soya saponin could increase the intestinal epithelial permeability, made the intestinal barrier weaker, and be considered to be the factor responsible for distal intestine enteritis induced by soybean meal used in Atlantic salmon (Salmo salar) ${ }^{[15]}$, juvenile Japanese flounder (Paralichthys 
olivaceus) ${ }^{[7]}$ and Zebra fish (Brachydanio rerio var ${ }^{[16]}$. Soy saponin may the main factor to restrict the capacity of using soybean meal in aquatic feed.

Rice field eel (Monopterus albus, M. albus) is subtropical freshwater benthic fish, mostly inhabit ponds, paddy fields, ditches and other water bodies, cave dwelling, day and night emergence, can endure low oxygen, feeding way for phagocytosis and swallowing, mainly by the way of phagocytosis ${ }^{[17]}$. M. albus is an economic species in central and southern of China, and popularly cage raised owing to its farming scale and commercial value ${ }^{[18]}$. M. albus requires high quality of protein level in diet referenced our previous studies ${ }^{[19,20]}$. Our published study reported that soybean meal ${ }^{[20]}$ and soy protein concentrate ${ }^{[21]}$ replaced FM decreased the growth performance on $M$. albus, and soy protein concentrate was better than soybean meal for $M$. albus. The digestive tract of $M$. albus is a long and coiled tube, and divided into four parts: mucosa, lamina propria-submucosa, muscularis and serosa. What's more, histology and cellular structures of epithelia and mucosa of the digestive tract of $M$. albus is alike other carnivorous fish ${ }^{[22]}$. Based on these characteristics of $M$. albus, in current study, we aimed to study the effects of soy isoflavone and soy saponin on intestinal structure and immunity in M. albus.

\section{Materials And Methods}

\subsection{Ingredients and experimental feeds}

Six isonitrogenous and isolipidic experimental feeds were made. A normal fish meal diet (550 g/ $\mathrm{kg}$ fish meal), a soybean meal diet (286 $\mathrm{g} / \mathrm{kg}$ fish meal; $372 \mathrm{~g} / \mathrm{kg}$ soybean meal) and a soy protein concentrate diet ( $286 \mathrm{~g} / \mathrm{kg}$ fish meal; $226 \mathrm{~g} / \mathrm{kg}$ soy protein concentrate) were set up according to our previous article ${ }^{[21]}$. Soy isoflavone (40\%) and soy saponin ( $\left.\geq 98 \%\right)$ and their mixed were supplemented with 2.5 $\mathrm{g} / \mathrm{kg}$ and $2 \mathrm{~g} / \mathrm{kg}$ in soy protein concentrate diet, respectively, purchased from Shanghai yuanye Bio-Technology Co.,Ltd. The levels of soy isoflavone and soy saponin were correspond to the soybean meal from previous study ${ }^{[23]}$, we have determined the level of soy isoflavone and soy saponin of soybean meal before making the experimental feeds. The composition of these diets and nutrition level were showed in Table 1. After crushing and sieving through 80 meshes, the feed material is evenly mixed according to the experimental formula. The feed material by air-dried at $25^{\circ} \mathrm{C}$ until the content of moisture is less than $10 \%$. Then it is stored in a refrigerator at $-20^{\circ} \mathrm{C}$ for reserve. Water was added before feeding and using as dough.

Table 1. Composition of the diets and nutrition level $(\mathrm{g} / \mathrm{kg})$ 


\begin{tabular}{|lllllll|}
\hline Ingredients & FM & SBM & SPC & SI & SS & SI+SS \\
\hline Fish meal & 550 & 286 & 286 & 286 & 286 & 286 \\
\hline Soy protein concentrate & 0 & 0 & 226 & 226 & 226 & 226 \\
\hline Soybean meal & 0 & 372 & 0 & 0 & 0 & 0 \\
\hline Wheat meal & 168 & 30 & 176 & 173.5 & 174 & 171.5 \\
\hline a-starch & 200 & 200 & 200 & 200 & 200 & 200 \\
\hline Brewer yeast & 50 & 50 & 50 & 50 & 50 & 50 \\
\hline Fish oil & 0 & 30 & 30 & 30 & 30 & 30 \\
\hline Choline chloride & 5 & 5 & 5 & 5 & 5 & 5 \\
\hline Soy isoflavone $(40 \%)$ & 0 & 0 & 0 & 2.5 & 0 & 2.5 \\
\hline Soy saponin $(\geq 98 \%)$ & 0 & 0 & 0 & 0 & 2 & 2 \\
\hline Ca( ${ }_{2}$ PO $\left.{ }_{4}\right)_{2}$ & 15 & 15 & 15 & 15 & 15 & 15 \\
\hline *Vitamin and Mineral premix & 12 & 12 & 12 & 12 & 12 & 12 \\
\hline Total & 1000 & 1000 & 1000 & 1000 & 1000 & 1000 \\
\hline Proximate analysis $(\mathrm{g} / \mathrm{kg})$ & & & & & & \\
\hline Dry matter & 901.31 & 898.68 & 897.52 & 898.91 & 899.47 & 902.15 \\
\hline Crude protein & 431.22 & 431.12 & 432.34 & 431.11 & 432.29 & 431.38 \\
\hline Crude lipid & 62.31 & 62.27 & 62.28 & 62.43 & 62.48 & 62.29 \\
\hline Crude ash & 101.11 & 100.91 & 99.97 & 99.82 & 100.01 & 99.86 \\
\hline Soy isoflavone & - & 1.00 & - & 0.99 & 0.01 & 1.00 \\
\hline Soy saponin & - & 2.00 & 0.01 & 0.01 & 2.01 & 2.01 \\
\hline
\end{tabular}

*Vitamin and Mineral premix view our previous study [24].

Feed samples analyzed according to AOAC ${ }^{[25]}$. Moisture was measured by drying to a constant weight at $105^{\circ} \mathrm{C}$ until it achieved a constant weight. Crude protein $(\mathrm{N} \times 6.25)$ was analyzed by the Kjeldahl method with an auto digestor (FOSS, Tecator, Hoganos, Sweden) after acid digestion. Crude lipid was determined by petroleum ether using the Soxtec System HT (Soxtec System HT6, Tecator, Sweden). Ash determined by a muffle furnace at $550{ }^{\circ} \mathrm{C}$ until complete ashing. Soy isoflavone and soy saponin of experimental diets were measured by high performance liquid chromatography (Agilent 1260, Agilent Technologies Co., Ltd., Santa Clara, CA, USA).

\subsection{Fish and experimental management}

M. Albus gained from Changde, China, raised in float cages $(2.0 \times 1.5 \times 1.5 \mathrm{~m})$ for 2 weeks to adapt. The fish were fed with earthworm a week, after that, the experimental feed of FM treatment was gradually added, the content of earthworm gradually decreased until the fish could completely consume the feed. The cages were cover by $95 \%$ of the fresh alternanthera philoxeroides (Mart.) Griseb to imitate the realistic living environment of $M$. albus.

Uniform size juveniles M. albus (initial weight $20.12 \pm 0.15 \mathrm{~g})$ randomized to 18 float cages $(2.0 \times 1.5 \times 1.5 \mathrm{~m})$ after fasting $24 \mathrm{~h}$. Every treatment consisted of triplicate groups with $80 \mathrm{fish}$, and fed apparent satiation once per day at 18:00 for 8 weeks. The feeding rate was $3-4 \%$ of body weight and adjusted according as the intake diet during the feeding trial (water temperature $28 \pm 4{ }^{\circ} \mathrm{C}$, dissolved $\mathrm{O}_{2} \geq 6.0$ $\mathrm{mg} / \mathrm{L}, \mathrm{NH}^{4+}-\mathrm{N} \otimes 0.5 \mathrm{mg} / \mathrm{L}$, respectively).

\subsection{Sample collection and analyses}


According to the guidelines established by the National Institutes of Health. All experimental fish anesthetized with eugenol $(1: 12,000)$ (Shanghai Reagent Corporation, Shanghai, China) before sampling to minimize suffering.

After feeding trial, M. albus were weighed and counted to calculate survival, weight gain and feed conversion ratio after fasting $24 \mathrm{~h}$. Hepatosomatic index, visceral somatic index and condition factor of $M$. albus were calculated based on the weight of liver, viscera, body and body length.

The caudal vein blood was heparinized from 5 fish each cage. Serum was obtained by centrifuging at $3500 \mathrm{~g}$ for 10 min, and being stored at $-80^{\circ} \mathrm{C}$ until using. Serum immunoglobulin $\mathrm{M}$, complement 3 and complement 4 were analyzed by a kit (Zhejiang Elikan Biological Technology Co., Ltd Wenzhou, Zhejiang, China). Serum acid phosphatase, alkaline phosphatase, glutamic pyruvic transaminase, glutamic oxalacetic transaminase, catalase, superoxide dismutase and total antioxidant capacity were measured by the kit of NanJing JianCheng Bioengineering (Nanjing, China).

Intestine was sampled from 5 fish each cage to measure the activity of trypsin, amylase, lipase, acid phosphatase and alkaline phosphatase by the kit of NanJing JianCheng Bioengineering (Nanjing, China). Intestinal immunoglobulin M, complement 3 and complement 4 were analyzed by a kit (Zhejiang Elikan Biological Technology Co., Ltd Wenzhou, Zhejiang, China).

Intestine from 3 fish in each cage were taken for histometric evaluation. Followed the processes of making slides, like washing, dehydrating by different grades of alcohol, clearing by xylene, and embedded in paraffin wax, sectioned wax blocks to five microns and stained with hematoxylin and eosin (H \& E) for histological slide. We used Olympus inverted microscope (IX53 Olympus inverted microscope, Olympus Co., Ltd, Tokyo, Japan) to observed the sections stained with H \& E of intestine. We have got nine photos of each treatment, based on these intestinal $\mathrm{H}$ \& E staining photos, we counted intestinal villus height, crypt depth, intestinal muscular thickness and amounts of intestinal goblet cells. The method referenced relative study of intestinal morphology on aquaculture animals ${ }^{\text {[26-28]. }}$

Intestinal total RNA was isolated by Trizol reagent (Invitrogen, USA). cDNA was synthesized by a SMART cDNA Synthesis Kit (Clontech Laboratories, Palo Alto, CA). The gene sequence obtained from National Center for Biotechnology Information, the primers were synthesised by Biosune Biotechnology, Inc. (Shanghai, China), which all indicated in Table 2. Using a BIO-RAD CFX96 touch quantitative Real-time PCR system (Applied Biosystems Inc., USA) to measure target gene expression. The method was referenced our previous article ${ }^{[29]}$. The amplification efficiency were between 0.95 and 1.00 , which calculated by the equation: $E=10 \star(-1 /$ slope $)-1,5$ fold serial dilutions of cDNA (triplicate) to make the standard curve. $2^{-\triangle \triangle C t}$ was used to calculate the expression of relative mRNA [30].

Table 2. Primer sequence for q-PCR 


\begin{tabular}{|c|c|c|}
\hline Gene & Primer & Accession NO. \\
\hline \multirow{2}{*}{${ }^{1} \mid \mathrm{IL}-1 \beta$} & F: 5'- GAGATGTGGAGCCCAAACTT-3' & KM113037.1 \\
\hline & R: 5'-CTGCCTCTGACCTTCTGGACTT-3' & \\
\hline \multirow[t]{2}{*}{${ }^{2} \mathrm{IL}-6$} & F: 5'-TGAGTGCCGACCCAGTTT-3' & XM_020606850.1 \\
\hline & R: 5'-CTTCAACCAGCCTATGGAGAC-3' & \\
\hline \multirow{2}{*}{${ }^{3} \mid \mathrm{L}-8$} & F: 5'-TACTGGTTCTGCTTACTGTCGC-3' & XM_020597092.1 \\
\hline & R: 5'-CAAATCTTTTGCCCATCCCT-3' & \\
\hline \multirow[t]{2}{*}{${ }^{4} \mid \mathrm{IL}-10$} & F: 5'-TTTGCCTGCCAAGTTATGAG-3' & XM_020593225.1 \\
\hline & R: 5'-CATTTGGTGACATCGCTCTT-3' & \\
\hline \multirow[t]{2}{*}{${ }^{5} \mathrm{IL}-12$} & F: 5'-CAAGTCAGTTGCCAAAATCC-3' & XM_020594580.1 \\
\hline & R: 5'-CCAAGCAGCTCAGGGTCT-3' & \\
\hline \multirow[t]{2}{*}{${ }^{6} \mathrm{IL}-15$} & F: 5'-AGAAATGCCCCATCTCCA-3' & XM_020622724.1 \\
\hline & R: 5'-CCCTGTCTCCGTCTTGTTG-3' & \\
\hline \multirow{2}{*}{${ }^{7} \mathrm{Tgfb} 1$} & F: 5'-AACCCACTACCTCACTACCCG-3' & XM_020623392.1 \\
\hline & R: 5'-GCCGAAGTTGGAAACCCT-3' & \\
\hline \multirow[t]{2}{*}{${ }^{8} \mathrm{Tgfb} 2$} & F: 5'-ATTACGCCAAGGAGGTGC-3' & XM_020622329.1 \\
\hline & R: 5'-GGGTTTTGAAGACGGAAGAT-3' & \\
\hline \multirow[t]{2}{*}{${ }^{9} \mathrm{Tgfb} 3$} & F: 5'-AGTTTGTCGCTATCCACTTGC-3' & XM_020590885.1 \\
\hline & R: 5'-GATGAGTTCCTTGGTGCTGTTA-3' & \\
\hline \multirow[t]{2}{*}{${ }^{10}{ }_{\mathrm{IFNy}}$} & F: 5'-GTCTGTCTGTCCCTCTGGCTAT-3' & NM_001360732.1 \\
\hline & R: 5'-TTGGGGTGGGCAGATTTT-3' & \\
\hline \multirow[t]{2}{*}{${ }^{11} \mathrm{NF}-\mathrm{kB}$} & F: 5'-ACCCTACCGTGACACTAACCT-3' & XM_020616319.1 \\
\hline & R: 5'-TGCCGTCTATCTTGTGGAAT-3' & \\
\hline \multirow[t]{2}{*}{${ }^{12}{ }_{\mathrm{IKBa}}$} & F: 5'-CСССТАССАССТСАССТАТG-3' & XM_020621992.1 \\
\hline & R: 5'-GTCCGTTCCACTCAAAATCA-3' & \\
\hline \multirow[t]{2}{*}{${ }^{13} \mathrm{IkBd}$} & F: 5'-CACTTGGCTGTAAAGGAGGG-3' & XM_020620120.1 \\
\hline & R: 5'-TGTTGCGGATGCTGGGAT-3' & \\
\hline \multirow[t]{2}{*}{${ }^{14}$ IKBe $_{\mathrm{KB}}$} & F: 5'-AGCACGGGCAGACAGAGT-3' & XM_020603944.1 \\
\hline & R: 5'-AGCCAAGTGAAGACAAGCAA-3' & \\
\hline \multirow[t]{2}{*}{${ }^{15} \mathrm{ZO}-1$} & F: 5'-GGCATCATCCCCAACAAA-3' & XM_020621576.1 \\
\hline & R: 5'-GCGAAGACCACGGAACCT-3' & \\
\hline \multirow[t]{2}{*}{${ }^{16} \mathrm{ZO}-2$} & F: 5'-AGCCGAGGTCGCACTTTA-3' & XM_020615114.1 \\
\hline & R: 5'-GCTTTGCTTCTGTGGTTGAT-3' & \\
\hline \multirow[t]{2}{*}{${ }^{17} \mathrm{CL}-12$} & F: 5'-TCACCTTCAATCGCAACG-3' & XM_020607277.1 \\
\hline & R: 5'-ATGTCTGGCTCAGGCTTATCT-3' & \\
\hline \multirow[t]{2}{*}{${ }^{18} \mathrm{CL}-15$} & F: 5'-CTCGCTGCTTGCTTTGACT-3' & XM_020611334.1 \\
\hline & R: 5'-TTGAAGGCGTACCAGGACA-3' & \\
\hline${ }^{19} \mathrm{OCC}$ & F: 5'-TGTCGGGGAGTGGGTAAA-3' & XM_020616177.1 \\
\hline
\end{tabular}

Page 6/28 


\begin{tabular}{|ll|} 
& \multicolumn{3}{|c|}{ R: 5'-TCCAGGCAAATAAAGAGGCT-3' } \\
${ }^{20}$ RPL17 & F: 5'-GTTGTAGCGACGGAAAGGGAC-3' $\quad$ XM_020587712.1 \\
& R: 5'-GACTAAATCATGCAAGTCGAGGG-3' \\
\hline
\end{tabular}

${ }^{1}$ IL-1 $\beta$ : Interleukin-1 $\beta$;

${ }^{2}$ IL-6: Interleukin-6;

${ }^{3} \mathrm{LL}-8$ : Interleukin-8;

${ }^{4}$ IL-10: Interleukin-10;

5IL-12: Interleukin-12;

${ }^{6}$ IL-15: Interleukin-15;

${ }^{7}$ Tgfb1: Transforming growth factor beta 1;

${ }^{8} \mathrm{Tgfb} 2$ : Transforming growth factor beta 2;

${ }^{9} \mathrm{Tgfb3}$ : Transforming growth factor beta 3;

${ }^{10}$ IFNY: Interferon gamma;

${ }^{11}$ NF-kB: NF-kB subunit;

${ }^{12}$ IKBa: NFKB inhibitor alpha;

${ }^{13} \mid$ KBd: NFKB inhibitor delta;

${ }^{14}$ IKBe: NFKB inhibitor epsilon;

${ }^{15}$ ZO-1: Tight junction protein ZO-1-like;

${ }^{16}$ ZO-2: Tight junction protein ZO-2-like;

${ }^{17} \mathrm{CL}-12$ : Claudin 12;

${ }^{18} \mathrm{CL}-15$ : Claudin 15;

${ }^{19}$ OCC: Occludin-like;

${ }^{20} \mathrm{RPL} 17$ is reference gene.

Gut content were sampled from two fish each cage, total six fish every treatment. DNA was extracted from the gut content by using a PowerFecal ${ }^{\text {TM }}$ DNA Isolation Kit (MoBio Laboratories, Inc), and quantified with a Nanodrop spectrophotometer (Thermo Scientific Inc. Waltham, MA, USA), used high-throughput sequencing by Illumina MiSeq platform. The method referenced our previous manuscript ${ }^{[17]}$. 2.4. Statistical analysis

Data were analysised by one-way analysis of variance (ANOVA) and outstanding differences among dietary groups were estimated by Duncan's multiple-range test. The results were described as means \pm S.E.M (standard error of the mean), outstanding level was chosen at $P<0.05$.

\section{Result}

3.1. Growth performance 
There has no significantly difference about survival rate and condition factor of $M$. albus among all treatments $(P>0.05)$. Compared with FM, the weight gain rate and viscerosomatic index of $M$. albus in SBM significantly decreased $(P<0.05)$, feed coefficient significantly increased $(P<0.05)$, and the growth of $M$. albus in SPC was better than that in SBM. Compared with SPC, dietary soy isoflavone and soy saponin (SI, SS, SI+SS) decreased growth of M. albus. (Table 3).

Table 3. Effects of dietary soy isoflavone and soy saponin on growth performance of M. Albus after 8 weeks.

\begin{tabular}{|llllllll|}
\hline Item & FM & SBM & SPC & SI & SS & SI+SS & $P$ value \\
\hline${ }^{1}$ IBW & $20.02 \pm 0.01$ & $20.01 \pm 0$ & $20.01 \pm 0.01$ & $20 \pm 0.01$ & $20.01 \pm 0.02$ & $20 \pm 0.01$ & 0.463 \\
\hline${ }^{2}$ FBW & $46.65 \pm 0.89^{\mathrm{b}}$ & $40.89 \pm 0.37^{\mathrm{a}}$ & $45.06 \pm 0.18^{\mathrm{ab}}$ & $43.88 \pm 0.34^{\mathrm{ab}}$ & $43.52 \pm 0.41^{\mathrm{ab}}$ & $42.59 \pm 0.25^{\mathrm{a}}$ & 0.015 \\
\hline${ }^{3} \mathrm{SR}$ & $96.25 \pm 1.44$ & $95 \pm 2.6$ & $95.42 \pm 2.32$ & $91.25 \pm 1.44$ & $95.42 \pm 1.82$ & $93.75 \pm 3.31$ & 0.379 \\
\hline${ }^{4} \mathrm{WG}$ & $133.03 \pm 4.39^{\mathrm{b}}$ & $104.39 \pm 1.79^{\mathrm{a}}$ & $125.14 \pm 0.9^{\mathrm{ab}}$ & $119.47 \pm 1.69^{\mathrm{ab}}$ & $117.48 \pm 2.16^{\mathrm{ab}}$ & $112.94 \pm 1.19^{\mathrm{a}}$ & 0.004 \\
\hline${ }^{5} \mathrm{FCR}$ & $1.74 \pm 0.06^{\mathrm{a}}$ & $2.22 \pm 0.04^{\mathrm{b}}$ & $1.85 \pm 0.01^{\mathrm{ab}}$ & $1.92 \pm 0.04^{\mathrm{ab}}$ & $1.97 \pm 0.04^{\mathrm{ab}}$ & $2.05 \pm 0.02^{\mathrm{b}}$ & 0.019 \\
\hline${ }^{6} \mathrm{HSI}$ & $6.98 \pm 0.16^{\mathrm{ab}}$ & $5.64 \pm 0.18^{\mathrm{a}}$ & $7.15 \pm 0.22^{\mathrm{b}}$ & $6.46 \pm 0.15^{\mathrm{ab}}$ & $5.89 \pm 0.28^{\mathrm{a}}$ & $5.65 \pm 0.14^{\mathrm{a}}$ & 0.013 \\
\hline${ }^{7} \mathrm{VSI}$ & $22.9 \pm 0.23^{\mathrm{b}}$ & $19.82 \pm 0.16^{\mathrm{a}}$ & $20.85 \pm 0.28^{\mathrm{ab}}$ & $22.42 \pm 0.34^{\mathrm{b}}$ & $19.79 \pm 0.24^{\mathrm{a}}$ & $19.01 \pm 0.18^{\mathrm{a}}$ & 0.018 \\
\hline${ }^{8} \mathrm{CF}$ & $10.66 \pm 0.1$ & $10.39 \pm 0.21$ & $10.82 \pm 0.14$ & $10.7 \pm 0.16$ & $10.97 \pm 0.19$ & $10.99 \pm 0.17$ & 0.146 \\
\hline
\end{tabular}

Values are presented as means \pm SEM $(n=3,80$ fish in each cage). Values in the same row with the same superscript or absence of superscripts are not significant different $(P>0.05)$.

${ }^{1}$ IBW: Initial body weight (g).

${ }^{2}$ FBW: Final body weight (g).

${ }^{3}$ SR: Survival rate $(\%)=100 \times$ final number of fish $/$ initial number of fish.

${ }^{4}$ Weight gain rate $(\%)=100 \times$ (final body weight - initial body weight) $/$ initial body weight.

${ }^{5}$ FCR: Feed conversion rate $(\%)=100 \times($ feed fed $/$ body weight gain $)$.

${ }^{6} \mathrm{HSI}$ : Hepatopancreas index $(\%)=100 \times$ (liver weight) $/$ (whole-body weight).

${ }^{7}$ VSI: Viscerosomatic index $(\%)=100 \times$ (viscera weight) $/$ (whole-body weight).

${ }^{8} \mathrm{CF}$ : Condition factor $(\%)=100 \times($ body weight $) /(\text { body length })^{3}$.

\subsection{Serum biochemical indices}

There was no significant difference in serum IgM and $\mathrm{C} 4$ among all groups $(P>0.05)$. Compared with FM, serum ACP, AKP, GOT, CAT were significantly increased in SBM $(P<0.05)$, while serum C3 significantly decreased in SBM $(P<0.05)$; and serum GOT significantly increased in SPC. Compared with SPC, serum AKP in SI significantly increased $(P<0.05)$. (Table 4).

Table 4. Effects of dietary soy isoflavone and soy saponin on serum biochemical indices of M. Albus after 8 weeks. 


\begin{tabular}{|c|c|c|c|c|c|c|c|}
\hline Item & FM & SBM & SPC & SI & SS & SI+SS & $P$ value \\
\hline${ }^{1} \mathrm{ACP}$ & $4.6 \pm 0.23^{a}$ & $6.25 \pm 0.15^{\mathrm{b}}$ & $5.78 \pm 0.13^{a b}$ & $6.13 \pm 0.25^{b}$ & $6.55 \pm 0.11^{b}$ & $6.51 \pm 0.18^{b}$ & 0.013 \\
\hline${ }^{2} \mathrm{AKP}$ & $0.97 \pm 0.04^{a}$ & $1.98 \pm 0.28^{b}$ & $1.55 \pm 0.13^{a b}$ & $2.31 \pm 0.19^{c}$ & $2.07 \pm 0.21^{b c}$ & $1.9 \pm 0.09^{a b}$ & 0.000 \\
\hline${ }^{3} \mathrm{GPT}$ & $2.02 \pm 0.08^{a}$ & $2.84 \pm 0.12^{a b}$ & $3.12 \pm 0.17^{a b}$ & $3.52 \pm 0.29^{b}$ & $3.99 \pm 0.15^{b}$ & $2.24 \pm 0.15^{\mathrm{a}}$ & 0.004 \\
\hline${ }^{4} \mathrm{GOT}$ & $1.62 \pm 0.06^{a}$ & $2.92 \pm 0.28^{b c}$ & $3.26 \pm 0.21^{c}$ & $3.59 \pm 0.32^{c}$ & $3.86 \pm 0.18^{c}$ & $2.8 \pm 0.3^{b}$ & 0.000 \\
\hline${ }^{5} \mathrm{CAT}$ & $1.62 \pm 0.08^{a}$ & $2.46 \pm 0.32^{b}$ & $1.85 \pm 0.12^{\mathrm{a}}$ & $1.95 \pm 0.14^{a b}$ & $1.85 \pm 0.08^{a}$ & $1.74 \pm 0.12^{\mathrm{a}}$ & 0.011 \\
\hline${ }^{6} \mathrm{SOD}$ & $1.34 \pm 0.09^{b}$ & $0.92 \pm 0.19^{a b}$ & $1.1 \pm 0.15^{\mathrm{ab}}$ & $1.06 \pm 0.2^{\mathrm{ab}}$ & $0.97 \pm 0.17^{a b}$ & $0.81 \pm 0.12^{a}$ & 0.017 \\
\hline${ }^{7} \mathrm{~T}-\mathrm{AOC}$ & $463.64 \pm 4.07^{a}$ & $467.35 \pm 3.75^{a}$ & $478.37 \pm 3.33^{a b}$ & $476.74 \pm 4.14^{\mathrm{ab}}$ & $498.79 \pm 5.45^{b}$ & $461.42 \pm 5.01^{a}$ & 0.016 \\
\hline${ }^{8} \lg M$ & $2.5 \pm 0.05$ & $2.48 \pm 0.04$ & $2.55 \pm 0.06$ & $2.7 \pm 0.09$ & $2.49 \pm 0.04$ & $2.49 \pm 0.07$ & 0.253 \\
\hline${ }^{9} \mathrm{C} 3$ & $1.17 \pm 0.23^{b}$ & $0.62 \pm 0.07^{a}$ & $0.98 \pm 0.1^{a b}$ & $1.21 \pm 0.25^{b}$ & $0.54 \pm 0.03^{a}$ & $0.64 \pm 0.05^{a}$ & 0.006 \\
\hline${ }^{10} \mathrm{C} 4$ & $0.24 \pm 0.02$ & $0.19 \pm 0.02$ & $0.27 \pm 0.02$ & $0.25 \pm 0.02$ & $0.21 \pm 0.02$ & $0.2 \pm 0.05$ & 0.225 \\
\hline
\end{tabular}

Values are presented as means \pm SEM $(n=3)$. Values in the same row with the same superscript or absence of superscripts are not significant different $(P>0.05)$.

${ }^{1}$ ACP: Acid phosphatase (King's unit/L)

${ }^{2}$ AKP: Alkaline phosphatase (King's unit/L)

${ }^{3} \mathrm{GPT}$ : Glutamic pyruvic transaminase (U/L)

${ }^{4}$ GOT: Glutamic oxalacetic transaminase (U/L)

${ }^{5}$ CAT: Catalase $(\mathrm{U} / \mathrm{mL})$

${ }^{6}$ SOD: Superoxide Dismutase (U/mg prot)

${ }^{7}$ T-AOC: Total antioxidant capacity (U/mL)

${ }^{8} \operatorname{IgM}:$ Immunoglobulin M (g/L)

${ }^{9} \mathrm{C} 3$ : Complement 3 (g/L)

${ }^{10} \mathrm{C} 4$ : Complement 4 (g/L)

\subsection{Activities of intestinal enzyme}

Compared with FM, intestinal ACP, AKP, C3, C4 and IgM remarkably declined in SBM $(P<0.05)$. Compared with SPC, intestinal IgM in SS remarkably declined $(P<0.05)$, and intestinal C3, C4 and IgM were significantly decreased in SI+SS $(P<0.05)($ Table 5$)$.

Table 5. Effects of dietary soy isoflavone and soy saponin on intestinal non-specific immune of M. Albus after 8 weeks. 


\begin{tabular}{|llllllll|}
\hline Item & FM & SBM & SPC & SI & SS & SI+SS & $P$ value \\
\hline${ }^{1}$ ACP & $21.04 \pm 0.82^{\mathrm{b}}$ & $14.93 \pm 0.6^{\mathrm{a}}$ & $18.03 \pm 0.79^{\mathrm{ab}}$ & $18.86 \pm 0.9^{\mathrm{ab}}$ & $18.22 \pm 0.86^{\mathrm{ab}}$ & $16.04 \pm 0.65^{\mathrm{a}}$ & 0.012 \\
\hline${ }^{2} \mathrm{AKP}$ & $18.65 \pm 0.57^{\mathrm{b}}$ & $13.28 \pm 0.74^{\mathrm{a}}$ & $15.9 \pm 1.06^{\mathrm{ab}}$ & $13.32 \pm 0.76^{\mathrm{a}}$ & $14.79 \pm 0.95^{\mathrm{ab}}$ & $14.59 \pm 0.65^{\mathrm{ab}}$ & 0.008 \\
\hline${ }^{3} \mathrm{C} 3$ & $1.03 \pm 0.02^{\mathrm{b}}$ & $0.83 \pm 0.04^{\mathrm{a}}$ & $1 \pm 0.03^{\mathrm{b}}$ & $0.9 \pm 0.07^{\mathrm{ab}}$ & $0.92 \pm 0.04^{\mathrm{ab}}$ & $0.88 \pm 0.04^{\mathrm{a}}$ & 0.025 \\
\hline${ }^{4} \mathrm{C} 4$ & $0.28 \pm 0.01^{\mathrm{b}}$ & $0.21 \pm 0.01^{\mathrm{a}}$ & $0.27 \pm 0.01^{\mathrm{b}}$ & $0.25 \pm 0.01^{\mathrm{ab}}$ & $0.25 \pm 0.01^{\mathrm{ab}}$ & $0.23 \pm 0.01^{\mathrm{a}}$ & 0.029 \\
\hline${ }^{5} \operatorname{lgM}$ & $2.01 \pm 0.12^{\mathrm{b}}$ & $1.42 \pm 0.07^{\mathrm{a}}$ & $1.94 \pm 0.16^{\mathrm{b}}$ & $1.66 \pm 0.09^{\mathrm{ab}}$ & $1.45 \pm 0.07^{\mathrm{a}}$ & $1.54 \pm 0.1^{\mathrm{a}}$ & 0.013 \\
\hline
\end{tabular}

Values are presented as means \pm SEM $(n=3)$. Values in the same row with the same superscript or absence of superscripts are not significant different $(P>0.05)$.

${ }^{1}$ ACP: Acid phosphatase (King's unit/mg protein)

${ }^{2}$ AKP: Alkaline phosphatase (King's unit/mg protein)

${ }^{3} \mathrm{C} 3$ : Complement 3 (g/mg protein)

${ }^{4} \mathrm{C} 4$ : Complement 4 (g/mg protein)

5IGM: Immunoglobulin M (g/mg protein)

\subsection{Activities of intestinal enzyme}

There have no significantly difference in amylase and lipase among all treatment $(P>0.05)$. Compared with FM, trypsin remarkably declined in SBM $(P<0.05)$. Compared with SPC, dietary soy isoflavone and soy saponin group (SI, SS, SI+SS) of trypsin were lower than SPC, not significantly $(P>0.05)$ (Table 6).

Table 6. Effects of dietary soy isoflavone and soy saponin on activities of intestinal digestive enzyme M. Albus after 8 weeks (U/mg protein).

\begin{tabular}{|llllllll|}
\hline Item & FM & SBM & SPC & SI & SS & SI+SS & $P$ value \\
\hline Amylase & $0.61 \pm 0.11$ & $0.74 \pm 0.03$ & $0.76 \pm 0.09$ & $0.67 \pm 0.04$ & $0.79 \pm 0.09$ & $0.72 \pm 0.07$ & 0.145 \\
\hline Lipase & $0.24 \pm 0.01$ & $0.26 \pm 0.02$ & $0.26 \pm 0.01$ & $0.25 \pm 0.01$ & $0.24 \pm 0.01$ & $0.27 \pm 0.01$ & 0.184 \\
\hline Trypsin & $2.62 \pm 0.03^{\mathrm{b}}$ & $1.59 \pm 0.04^{\mathrm{a}}$ & $1.99 \pm 0.02^{\mathrm{ab}}$ & $1.36 \pm 0.03^{\mathrm{a}}$ & $1.34 \pm 0.02^{\mathrm{a}}$ & $1.64 \pm 0.02^{\mathrm{ab}}$ & 0.016 \\
\hline
\end{tabular}

Values are presented as means \pm SEM $(n=3)$. Values in the same row with the same superscript or absence of superscripts are not significant different $(P>0.05)$.

\subsection{The sections stained with $\mathrm{H} \& \mathrm{E}$ of intestine}

The results of intestinal H \& E staining showed in figure 1. Based on the intestinal H \& E staining photos, intestinal villus height, crypt depth, intestinal muscular thickness and amounts of intestinal goblet cells were been counted and showed in table 7. Compared with FM, intestinal villus height and intestinal muscular thickness in SBM were remarkably decreased $(P<0.05)$, intestinal crypt depth in SBM was remarkably increased $(P<0.05)$. Compared with SPC, the trend showed that intestinal crypt depth in dietary soy isoflavone and soy saponin (SI, SS, SI+SS) were increased, while intestinal villus height in SI, SS, SI+SS were decreased, amounts of intestinal goblet cells per root in SI+SS was remarkably declined.

Table 7. Effects of dietary soy isoflavone and soy saponin on the intestinal sections stained with $\mathrm{H} \& \mathrm{E}$ (100 times and 400 times) of $M$. Albus after 8 weeks $(\mathrm{n}=3)$. 


\begin{tabular}{|llllllll|}
\hline Item & FM & SBM & SPC & SI & SS & SI+SS & $P$ value \\
\hline${ }^{1}$ IVH & $47.37 \pm 4.27^{\mathrm{b}}$ & $31.87 \pm 2.4^{\mathrm{a}}$ & $40.37 \pm 0.4^{\mathrm{ab}}$ & $36.41 \pm 2.6^{\mathrm{ab}}$ & $34.09 \pm 2.72^{\mathrm{a}}$ & $37.14 \pm 3.23^{\mathrm{ab}}$ & 0.018 \\
\hline${ }^{2} \mathrm{CD}$ & $1.37 \pm 0.09^{\mathrm{a}}$ & $2.27 \pm 0.22^{\mathrm{b}}$ & $1.38 \pm 0.06^{\mathrm{a}}$ & $1.73 \pm 0.15^{\mathrm{ab}}$ & $1.75 \pm 0.06^{\mathrm{ab}}$ & $1.96 \pm 0.29^{\mathrm{ab}}$ & 0.006 \\
\hline${ }^{3} \mathrm{IMT}$ & $3.46 \pm 0.2^{\mathrm{b}}$ & $2.6 \pm 0.22^{\mathrm{a}}$ & $3.17 \pm 0.42^{\mathrm{ab}}$ & $2.87 \pm 0.1^{\mathrm{a}}$ & $3.24 \pm 0.09^{\mathrm{b}}$ & $2.62 \pm 0.14^{\mathrm{a}}$ & 0.011 \\
${ }^{4} \mathrm{AIGC}$ & $38.33 \pm 4.91^{\mathrm{ab}}$ & $26.67 \pm 2.91^{\mathrm{a}}$ & $42 \pm 0^{\mathrm{b}}$ & $44.67 \pm 17.48^{\mathrm{b}}$ & $33.67 \pm 2.96^{\mathrm{ab}}$ & $29.67 \pm 2.4^{\mathrm{a}}$ & 0.002 \\
\hline
\end{tabular}

Values are presented as means \pm SEM $(n=3)$. Values in the same row with the same superscript or absence of superscripts are not significant different $(P>0.05)$.

${ }^{1} \mathrm{IVH}$ : Intestinal villus height $(\mu \mathrm{m})$

${ }^{2} \mathrm{CD}$ : Crypt depth $(\mu \mathrm{m})$

${ }^{3} \mathrm{IMT}:$ Intestinal muscular thickness ( $\left.\mu \mathrm{m}\right)$

${ }^{4}$ AIGC: Amounts of intestinal goblet cells per root

3.6. Intestinal regulatory mRNA expression

Compared with FM, OCC, CL12, ZO-1, ZO-2, IL-10, Tgfb1, Tgfb2, IkBa and IkBe mRNA expression significantly down regulated in SBM ( $P$ $<0.05)$, IL-1 $\beta$, IL-8, IL-12, IL-15, IFNy mRNA expression significantly up regulated in SBM $(P<0.05)$; ZO-1, Tgfb1, NF-KB mRNA expression significantly down regulated in SPC $(P<0.05)$. Compared with SPC, CL-12, IL-15, ZO-2, IL-10, Tgfb2 mRNA expression down regulated in dietary soy isoflavone and soy saponin groups (SI, SS, SI+SS), while IL-1 $\beta$, IL-6, IL-8, IL-12 and IL-15 mRNA expression up regulated in these groups (Figure 2).

\subsection{OUT number of bacterial diversity index}

OTU numbers were ranked as SS $>$ SPC $>\mathrm{FM}>\mathrm{SI}>\mathrm{SS}+\mathrm{SI}>\mathrm{SBM}$ from sequence 0 to 60000 until stability, the OTU number of all groups were increased if sequences number is under 2609, the OTU Number in SS was increased the fastest among all groups, which in SBM was the lowest (Figure 3).

\subsection{Bacterial diversity index based on 16S rDNA gene sequence}

Good's estimated sample coverage (ESC) in all groups were higher than $99 \%$, which indicated that the data covered most of bacterial species. Compared with FM, shannon, simpson, chao1, abundance based coverage estimator (ACE) and operational taxonomic units (OTUs) remarkably decreased in SBM $(P<0.05)$, these indices in SPC also decreased, not significantly. Compared in SPC, shannon significantly decreased in SI+SS $(P<0.05)$ (Table 8).

Table 8. Effects of dietary soy isoflavone and soy saponin on bacterial diversity indexes based on 16s rDNA gene sequence after feeding 8 weeks $(n=3)$. 


\begin{tabular}{|llllllll|}
\hline Item & FM & SBM & SPC & SI & SS & SI+SS & $\begin{array}{c}P \\
\text { value }\end{array}$ \\
\hline${ }^{1}$ ESC & $0.99 \pm 0.02$ & $0.98 \pm 0.01$ & $0.98 \pm 0.01$ & $0.99 \pm 0.01$ & $0.99 \pm 0.02$ & $0.99 \pm 0.03$ & 0.577 \\
\hline Shannon & $4.74 \pm 0.46^{\mathrm{b}}$ & $3.53 \pm 0.06^{\mathrm{a}}$ & $4.52 \pm 0.81^{\mathrm{b}}$ & $4.08 \pm 0.14^{\mathrm{ab}}$ & $4.46 \pm 0.66^{\mathrm{ab}}$ & $3.92 \pm 0.77^{\mathrm{a}}$ & 0.017 \\
\hline Simpson & $0.91 \pm 0.02$ & $0.83 \pm 0.03$ & $0.88 \pm 0.07$ & $0.82 \pm 0.04$ & $0.86 \pm 0.06$ & $0.82 \pm 0.07$ & 0.346 \\
\hline Chao1 & $430.99 \pm 55.16^{\mathrm{b}}$ & $301.58 \pm 27.31^{\mathrm{a}}$ & $396.78 \pm 39.99^{\mathrm{ab}}$ & $388.73 \pm 3.38^{\mathrm{ab}}$ & $411.68 \pm 38^{\mathrm{ab}}$ & $344.53 \pm 36.69^{\mathrm{ab}}$ & 0.003 \\
\hline${ }^{2}$ ACE & $441.03 \pm 52.72^{\mathrm{b}}$ & $296.71 \pm 32.83^{\mathrm{a}}$ & $381.33 \pm 50.33^{\mathrm{ab}}$ & $403.21 \pm 6.85^{\mathrm{ab}}$ & $425.31 \pm 38.15^{\mathrm{ab}}$ & $345.04 \pm 38.7^{\mathrm{ab}}$ & 0.000 \\
\hline${ }^{3}$ OTUs & $372.33 \pm 62.5^{\mathrm{b}}$ & $210.67 \pm 32.59^{\mathrm{a}}$ & $316 \pm 58.04^{\mathrm{ab}}$ & $326.33 \pm 17.19^{\mathrm{ab}}$ & $326.33 \pm 61.5^{\mathrm{ab}}$ & $247 \pm 22.91^{\mathrm{ab}}$ & 0.005 \\
\hline
\end{tabular}

Values are presented as means \pm SEM $(n=3)$. Values in the same row with the same superscript or absence of superscripts are not significant different $(P>0.05)$.

${ }^{1}$ ESC: Good's estimated sample coverage

${ }^{2} \mathrm{ACE}:$ abundance-based coverage estimator

${ }^{3}$ OTUs: Operational taxonomic units

\subsection{Dominant bacteria and relative abundance}

As figure 4 showed, firmicutes were the most abundant groups of gut flora of $M$. albus. For the level of phylum, compared with FM, abundance of firmicutes were trended to increase in SBM and SPC, firmicutes in SBM was the highest, while proteobacteria were trended to decrease in SBM and SPC, proteobacteria in SBM was the lowest. Compared with SPC, abundance of firmicutes were trended to increase in SS and SI+SS, proteobacteria were trended to decrease in dietary soy isoflavone and soy saponin groups (SI, SS, SI+SS). As figure 5 showed, compared with FM, ratio of gut stenotrophomons in SBM and SPC decreased, and SBM was the lowest; ratio of gut lactococcus in SBM and SPC increased, ratio of unidentified_clostridiales was the highest in SBM. Compared with SPC, romboutsia, unidentified_ clostridiales and stenotrophomons increased in dietary soy isoflavone and soy saponin groups (SI, SS, SI+SS), while cetobacterium decreased in dietary soy isoflavone and soy saponin groups (SI, SS, SI+SS). Dietary soy isoflavone and soy saponin group were different from the SPC group, and SI+SS group was quietly (Figure 6).

\section{Discussion}

This study was continuously explore soy protein replace fish meal on M. albus, and referenced our previous studies. In this study, the growth performance of M. albus in SBM remarkably lower than FM, while SPC group not remarkably decreased, and growth performance of $M$. albus in dietary soy isoflavone and soy saponin group (SI, SS, SI+SS) were lower than SPC. Here, dietary soy isoflavone and soy saponin also decreased the hepatopancreas index and viscerosomatic index of $M$. albus, in particular soy isoflavone and soy saponin exist simultaneously. This phenomenon was similar to study that high level of soy saponin decreased growth performance on juvenile Japanese flounder (Paralichthys olivaceus) ${ }^{[7]}$. While dietary soy isoflavone has the negatively affect growth performance on Atlantic salmon (Salmo salar L.) ${ }^{[12]}$, dietary soy isoflavone not significantly influence growth performance on rainbow trout (Oncorhynchus mykiss) ${ }^{[8]}$. However, dietary higher soy isoflavone showed better growth than without supplemented soy isoflavone on juvenile golden pompano (Trachinotus ovatus) ${ }^{[31]}$ and juvenile grass carp (Ctenopharyngodon idella) ${ }^{[11]}$. We speculated that different aquatic animals have different tolerance to different level of soy isoflavone and soy saponin. In our study, dietary level of soy isoflavone and soy saponin which corresponded to the soybean meal were all decreased the growth performance of $M$. albus, and these two heat-stable anti-nutritional factors have negative stacking effect for growth performance on M. albus.

Acid phosphatase (ACP) enzyme is involved in protein pinocytosis and intracellular digestion ${ }^{[32]}$. Alkaline phosphatase (AKP) is key enzyme with protective role in fish under stress, parasitic infection and wound healing ${ }^{[33]}$, and it also play a main role in dephosphorylating and detoxifying the endotoxin components of lipopolysaccharides in the intestinal mucosa ${ }^{[34]}$. Transaminases are 
produced by liver, if the liver damaged, the permeability of the cell membrane increases, and transaminases been secreted from cells, causing thus transaminase higher in blood. Glutamic oxalacetic transaminase (GOT) and glutamic pyruvic transaminase (GPT) used to evaluate liver whether injury ${ }^{[35]}$. Complement system is considered that defense system for resistance against microbial infection, mainly included C3 and C4 ${ }^{[36]}$. Superoxide dismutase (SOD) can eliminate free radicals, like hydroxyl radicals and superoxide anion radicals, improve antioxidant capacity ${ }^{[37]}$. Immunoglobulin $\mathrm{M}(\mathrm{IgM})$ is the essential immunoglobulin and sensitively react xenobiotics, used as an important biomarker for humoral adaptive immune response in fish ${ }^{[38]}$. Total antioxidant capacity (T-AOC) is an important indicator to measured the antioxidant ability of the body, previous studies hold that soy isoflavone and soy saponin are antioxidant factor ${ }^{[10,14]}$. In present study, compared with SPC, serum AKP was remarkably increased in SI, serum GOT in dietary soy isoflavone and saponin group were significantly increased, but serum SOD were showed the decreasing trend in dietary soy isoflavone and soy saponin groups. On the one hand, dietary soy isoflavone and soy saponin have antioxidant capacity for $M$. albus, this phenomenon was similar to juvenile grass carp (Ctenopharyngodon idella) ${ }^{[11]}$. On the other hand, these anti-nutrition factors also damaged the liver of $M$. albus. What's more, we also determined intestinal non-specific immune indices. Here, compared with SPC, intestinal IgM in SS group was remarkably declined, intestinal C3, C4 and IgM also significantly decreased in SI+SS group. We hold that soy saponin decreased the intestinal immunity, and soy isoflavone and soy saponin had the stacking effect on $M$. albus. While there have dietary soy isoflavone improved the immune response on juvenile golden pompano (Trachinotus ovatus) ${ }^{[31]}$ and juvenile grass carp (Ctenopharyngodon idella) ${ }^{[11]}$. May be different types of fish have varying degrees of tolerance by soy isoflavone and soy saponin.

In this work, activity of intestinal trypsin enzyme in SBM group were remarkably lower than FM group, dietary soy isoflavone and soy saponin and its mixed decreased trypsin. M. albus is a kind of carnivorous aquatic animal [39], higher activity of trypsin is benefit for protein digestion ${ }^{[24]}$. These results may explain that dietary soy isoflavone and soy saponin decreased growth of M. albus. While our results was opposite to the study that dietary soy isoflavone increased intestinal digestive of trypsin on Atlantic salmon (Salmo salarL.) [12]. The specific reasons need further exploring.

In order to observe dietary soy isoflavone and soy saponin on intestinal tract more intuitively, we had made the intestinal $\mathrm{H}$ \& E staining. Based on these intestinal morphology photos, we have counted intestinal villus height, crypt depth, intestinal muscular thickness and amount of intestinal goblet cells. In this study, compared with FM, intestinal villus height and intestinal muscular thickness in SBM remarkably decreased, intestinal crypt depth in SBM was increased, and these indices had the same phenomenon in SPC, but not remarkably. Compared with SPC, the trend showed that intestinal crypt depth increased, while intestinal villus height in dietary soy isoflavone and soy saponin (SI, SS, SI+SS) were decreased, amounts of intestinal goblet cells per root in SI+SS was remarkably declined. It well explain that dietary soy isoflavone and soy saponin decreased intestinal trypsin and immunity, also decreased the abilities of intestinal digestion and absorption, thus made the negative effect on performance of $M$. albus. These results were similar to the study on juvenile Japanese flounder (Paralichthys olivaceus) ${ }^{[7]}$, Zebrafish (Brachydanio rerio var) ${ }^{[4]}$, Atlantic salmon (Salmo salar L.) ${ }^{[12]}$ and juvenile turbot (Scophthalmus maximus) ${ }^{[6]}$. Soy saponin could increase the intestinal epithelial permeability ${ }^{[40]}$, and made the intestinal cells easily damaged, and soy isoflavone promote this process, thus the function of intestinal barrier decreasing. In contrast to our results, dietary soy isoflavone didn't affected intestinal structure of Rainbow trout (Oncorhynchus mykiss) ${ }^{[8]}$.

To further exploring effects of dietary soy isoflavone and soy saponin intestinal immune and its mechanism, we have intestinal barrier and immune mRNA expression. The intestinal tight junction protein, include occludens, claudin and zonula, which are indispensable in protecting barrier integrity and function, they play an epithelial barrier and prevented infiltration by various viruses and antigens ${ }^{[41]}$. Tight junction proteins would damage the intestinal permeability if they mRNA expression declined. Much research proved that IL-1 $\beta$, IL12 , IL-15,IL-8 and IFNy were the key pro-inflammatory cytokines ${ }^{[22-44]}$, and IL-10 and Tgfb mainly prevent inflammatory occuring ${ }^{[45]}$. When intestinal tight junction damaged, the risk of intestinal inflammation elevates, intestinal inflammation mediated by antiinflammatory cytokines and pro-inflammatory cytokines, and pro-inflammatory cytokines would promote the differentiation of $\mathrm{T}$ cells and $B$ cells and make inflammatory occuring ${ }^{[46]}$. Then, IL-1 $\beta$, IL-12 and IFNץ mRNA expression up-regulated mean that inflammation has occurred. On contrary to pro-inflammatory cytokines, the anti-inflammatory cytokines could reduce the risk of cytokines. For example, Tgfb could inhibit the lymphocyte proliferation ${ }^{[47]}$. NF-KB has been demonstrated that the most important regulators of inflammatory gene expression ${ }^{[48]}$. After the stimulation of pro-inflammatory pathways, the IKB would be degraded, and subsequently activate the translocation of NF-KB into the nucleus, which regulated the expression of pro-inflammatory cytokines and caused the inflammatory responses ${ }^{[49]}$. Soybean saponins induced NF-KB by blocking IKBa degradation. The current study was different with the published study that soybean saponins exhibit anti-inflammatory by suppressed inflammatory cytokine genes transcription through the NF-KB signaling pathway in mice ${ }^{[50]}$. In present study, compared with FM, intestinal tight junction protein mRNA (OCC, CL12, ZO-1, ZO-2, 
IL-10) down regulated in SBM, expression of anti-inflammatory factors mRNA (Tgfb1 and Tgfb2) and inflammatory signaling molecules mRNA (IKBa and IKBe) significantly down regulated in SBM, pro-inflammatory factors mRNA (IL-13, IL-8, IL-12, IL-15, IFNץ) remarkably up regulated in SBM, ZO-1, Tgfb1, NF-KB mRNA expression significantly down regulated in SPC. Dietary soy isoflavone and soy saponin down regulated CL-12, IL-15, ZO-2, IL-10, Tgfb2 mRNA expression, while IL-1 $\beta$, IL-6, IL-8, IL-12 and IL-15 mRNA expression up regulated in these groups. Our findings were similar to study in Atlantic salmon (Salmo salarL.) ${ }^{[40]}$. It suggested that soy saponin increased the intestinal permeability, damaged the intestinal structure and caused the function of intestinal barrier decreasing, and soy isoflavone promote this progress, eventually, made the intestinal inflammation of $M$. albus easily occurred. A paper also reported that dietary soy saponin made higher expression of intestinal IL-1 $\beta$ and IL-8 on juvenile turbot (Scophthalmus maximus), and damaged the intestinal barrier and induced enteritis ${ }^{[6]}$. While dietary soy isoflavone improved immune performance (such as the responses of immune cytokines) of juvenile grass carps ${ }^{[11]}$. We think that herbivorous fish may have higher adaptability to plant anti-nutritional factors than carnivorous fish.

As we know, healthy gut microbiota is essential to promote host intestinal structure and maintain it healthy statement ${ }^{[51]}$. Our previous studies have demonstrated that diet could change the composition of gut bacterial communities of $M$. albus ${ }^{[17,24,52]}$. In current study, OTU numbers ranked as SS > SPC > FM > SI > SS+SI > SBM from sequence 0 to 60000 until stability, Good's estimated sample coverage (ESC) in all treatments were higher than $99 \%$, which indicated that our data is credible. Compared with FM, shannon, simpson, chao1, abundance based coverage estimator (ACE) and operational taxonomic units (OTUs) were remarkably decreased in SBM, these indices in SPC also decreased, not significantly. And shannon significantly lower in SI+SS than SPC. This phenomenon may indicate that dietary soy isoflavone and soy saponin made the gut flora imbalance. For the level of phylum, firmicutes were the most abundant group of M. albus, compared with FM, abundance of firmicutes were trended to increase in SBM and SPC, firmicutes in SBM was the highest, while proteobacteria were trended to decrease in SBM and SPC, proteobacteria in SBM was the lowest. Compared with SPC, gut firmicutes were trended to increase in SS and SI+SS, proteobacteria were trended to decrease in dietary soy isoflavone and soy saponin groups (SI, SS, SI+SS). For the level of genus, compared with FM, ratio of gut stenotrophomons in SBM and SPC decreased, and SBM was the lowest; ratio of gut lactococcus in SBM and SPC increased, ratio of unidentified_clostridiales was the highest in SBM. Compared with SPC, romboutsia, unidentified_ clostridiales and stenotrophomons increased in dietary soy isoflavone and soy saponin groups (SI, SS, SI+SS), while cetobacterium decreased in dietary soy isoflavone and soy saponin groups (SI, SS, SI+SS). Firmicutes were the most abundant groups of phylum in the gut bacteria of M.albus, which was similar to our previous results ${ }^{[24]}$, it is also key microbiota to affect immune ${ }^{[53]}$, it's an good evidence to explain that dietary soy isoflavone and soy saponin mixed feed caused immunity declining.

\section{Conclusion}

Soy isoflavone and soy saponin could damage the intestinal structure and change gut bacteria community of $M$. albus, thus destroyed intestinal barrier, eventually induced intestinal inflammatory occurring, also made the negative effect on growth performance. Dietary soy isoflavone and soy saponin mixed feed has more serious effect than they single supplemented.

\section{Declarations}

\section{Ethics approval and consent to participate}

All experiments involving animals were supported by the Animal Care Committee of Hunan Agricultural University (Changsha, Hunan Province, China) and were conducted according to the Chinese guidelines for animal welfare.

\section{Consent for publication}

Not applicable.

\section{Availability of data and materials}

The datasets used and/or analysed during the current study are available from the corresponding author on reasonable request.

\section{Competing interests}

The authors declare that they have no competing interests. 


\section{Funding}

This study was financially supported by National Natural Science Foundation of China (Grant No. 31572626).

\section{Authors' contributions}

Yajun Hu determined the index in of the study and wrote this article.

Wuying Chu was in charge of designing gene in this trial.

Junzhi Zhang participated in experimental designing for this study.

Yi Hu participated in experimental designing and revised this manuscript.

\section{Acknowledgments}

Not applicable.

\section{References}

1. TACON A G J, METIAN M. Global overview on the use of fish meal and fish oil in industrially compounded aquafeeds: Trends and future prospects[J]. Aquaculture, 2008,285(1-4): 146-158.

2. GATLIN D M, BARROWS F T, BROWN P, et al. Expanding the utilization of sustainable plant products in aquafeeds: a review[J]. Aquaculture Research, 2007,38(6): 551-579.

3. ZHOU Z, RINGØE, OLSEN R E, et al. Dietary effects of soybean products on gut microbiota and immunity of aquatic animals: A review[J]. Aquaculture Nutrition, 2018,24(1): 644-665.

4. HEDRERA M I, GALDAMES J A, JIMENEZ-REYES M F, et al. Soybean Meal Induces Intestinal Inflammation in Zebrafish Larvae[J]. PLoS ONE, 2013,8(7): e69983.

5. ARI M M, AYANWWALE B A, ADAMA T Z, et al. Evaluation of the chemical composition and anti nutritional factors (ANFs) levels of different thermally processed soybeans[J]. Asian Journal of Agriculture Research, 2012,6(2): 91-98.

6. GU M, JIA Q, ZHANG Z, et al. Soya-saponins induce intestinal inflammation and barrier dysfunction in juvenile turbot (Scophthalmus maximus)[J]. Fish \& Shellfish Immunology, 2018,77: 264-272.

7. CHEN W, AI Q, MAI K, et al. Effects of dietary soybean saponins on feed intake, growth performance, digestibility and intestinal structure in juvenile Japanese flounder (Paralichthys olivaceus)[J]. Aquaculture, 2011,318(1-2): 95-100.

8. PASTORE M R, NEGRATO E, POLTRONIERI C, et al. Effects of dietary soy isoflavones on estrogenic activity, cortisol level, health and growth in rainbow trout, Oncorhynchus mykiss[J]. Aquaculture Research, 2018,49(4): 1469-1479.

9. MASILAMANI M, WEI J, SAMPSON H A. Regulation of the immune response by soybean isoflavones[J]. Immunologic research, 2012,54(1-3): 95-110.

10. CHEN X R, TAN B P, MAI K S, et al. Dietary administration of soybean isoflavones enhances the immunity of white shrimp Litopenaeus vannamei and its resistance against Vibrio alginolyticus[J]. Aquaculture Nutrition, 2011,17(1): 24-32.

11. CAO S, XIONG D, LUO W, et al. Effects of dietary soy isoflavones on growth, antioxidant status, immune response and resistance of juvenile grass carp (Ctenopharyngodon idella) to Aeromonas hydrophila challenge[J]. Aquaculture Research, 2020,51(6): 24722482.

12. GU M, GU J N, PENN M, et al. Effects of diet supplementation of soya-saponins, isoflavones and phytosterols on Atlantic salmon (Salmo salar, L) fry fed from start-feeding[J]. Aquaculture Nutrition, 2015,21(5): 604-613.

13. TARADE K M, SINGHAL R S, JAYRAM R V, et al. Kinetics of degradation of saponins in soybean flour (Glycine max.) during food processing[J]. Journal of Food Engineering, 2006,76(3): 440-445.

14. FRANCIS G, KEREM Z, MAKKAR H P S, et al. The biological action of saponins in animal systems: a review[J]. British Journal of Nutrition, 2002,88(6): 587-605.

15. GU M, KORTNER T M, PENN M, et al. Effects of dietary plant meal and soya-saponin supplementation on intestinal and hepatic lipid droplet accumulation and lipoprotein and sterol metabolism in Atlantic salmon (Salmo salar L.)[J]. British Journal of Nutrition, 2014,111(03): 432-444. 
16. FUENTES-APPELGREN P, OPAZO R, BARROS L, et al. Effect of the Dietary Inclusion of Soybean Components on the Innate Immune System in Zebrafish[J]. Zebrafish, 2014,11(1): 41-49.

17. PENG M, XUE J, HU Y, et al. Disturbance in the homeostasis of intestinal microbiota by a high-fat diet in the rice field eel (Monopterus albus)[J]. Aquaculture, 2018,502(2019): 347-355.

18. HU Y, ZHANG J, HE L, et al. Effects of dietary vitamin C on growth, antioxidant activity, and immunity in rice field eel (Monopterus albus)[J]. Journal of the World Aquaculture Society, 2019,51(1): 159-170.

19. MA X, HU Y, WANG X, et al. Effects of practical dietary protein to lipid levels on growth, digestive enzyme activities and body composition of juvenile rice field eel (Monopterus albus)[J]. Aquaculture International, 2014,22(2): $749-760$.

20. HU Y, YANG G, LI Z, et al. Effect of dietary taurine supplementation on growth, digestive enzyme, immunity and resistant to dry stress of rice field eel (Monopterus albus) fed low fish meal diets[J]. Aquaculture Research, 2018,49(6): 2108-2118.

21. ZHANG J, ZHONG L, PENG M, et al. Replacement of fish meal with soy protein concentrate in diet of juvenile rice field eel Monopterus albus[J]. Aquaculture Reports, 2019,15(2019): 100235.

22. DAI X, SHU M, FANG W. Histological and ultrastructural study of the digestive tract of rice field eel, Monopterus albus[J]. Journal of Applied Ichthyology, 2007,23(2): 177-183.

23. SILVA F D O, PERRONE D. Characterization and stability of bioactive compounds from soybean meal[J]. LWT- Food Science \& Technology, 2015,63(2): 992-1000.

24. HU Y, HUANG Y, TANG T, et al. Effect of partial black soldier fly (Hermetia illucens L.) larvae meal replacement of fish meal in practical diets on the growth, digestive enzyme and related gene expression for rice field eel (Monopterus albus)[J]. Aquaculture Reports, 2020,17: 100345.

25. AOAC. Association of Official Analytical Chemists Official. Methods of Analysis, 16th edition. AOAC, Arlington, Virginia, USA.[J]. 1995.

26. PENG M, XU W, Al Q, et al. Effects of nucleotide supplementation on growth, immune responses and intestinal morphology in juvenile turbot fed diets with graded levels of soybean meal (Scophthalmus maximus L.)[J]. Aquaculture, 2013,392-395: 51-58.

27. RAMOS M A, BATISTA S, PIRES M A, et al. Dietary probiotic supplementation improves growth and the intestinal morphology of Nile tilapia[J]. animal, 2017,11(8): 1259-1269.

28. TANG T, ZHONG L, YU D, et al. Evaluation of dried porcine solubles in diets of Rice field eel (Monopterus albus)[J]. Aquaculture, 2021,531: 735897.

29. HU Y, HU Y, WU T, et al. Effects of High Dietary Levels of Cottonseed Meal and Rapeseed Meal on Growth Performance, Muscle Texture, and Expression of Muscle-Related Genes in Grass Carp[J]. North American Journal of Aquaculture, 2019,81(3): $235-241$.

30. LIVAK, J K, SCHMITTGEN, et al. Analysis of Relative Gene Expression Data Using Real-Time Quantitative PCR and the 2- $\triangle \triangle C T$ Method[J]. Methods, 2001,25(4): 402-408.

31. ZHOU C, LIN H, GE X, et al. The Effects of dietary soybean isoflavones on growth, innate immune responses, hepatic antioxidant abilities and disease resistance of juvenile golden pompano Trachinotus ovatus[J]. Fish \& Shellfish Immunology, 2015,43(2015): 158-166.

32. NIGAM A K, KUMARI U, MITTAL S, et al. Comparative analysis of innate immune parameters of the skin mucous secretions from certain freshwater teleosts, inhabiting different ecological niches[J]. Fish Physiology and Biochemistry, 2012,38(5): 1245-1256.

33. PALAKSHA K J, SHIN G, KIM Y, et al. Evaluation of non-specific immune components from the skin mucus of olive flounder (Paralichthys olivaceus)[J]. Fish \& Shellfish Immunology, 2008,24(4): 479-488.

34. BATES J M, AKERLUND J, MITTGE E, et al. Intestinal Alkaline Phosphatase Detoxifies Lipopolysaccharide and Prevents Inflammation in Zebrafish in Response to the Gut Microbiota[J]. Cell host \& microbe, 2007,2(6): 371-382.

35. HYDER M A, HASAN M, MOHIELDEIN A H. Comparative Levels of ALT, AST, ALP and GGT in Liver associated Diseases[J]. European Journal of Experimental Biology, 2013,3(2): 280-284.

36. XIN Y, HERTLE E, van der KALLEN C J H, et al. Complement C3 and C4, but not their regulators or activated products, are associated with incident metabolic syndrome: the CODAM study[J]. Endocrine, 2018,62(3): 617-627.

37. RADMILA C, PAVLE S, DEAN J, et al. The influence of nutrition (diet treatment) in streptozotocin - Induced diabetic rats[J]. Macedonian Veterinary Review, 2013,36(1): 41-47.

38. LIM S, HO H, KHADER S M S, et al. First report on the isolation of immunoglobulin M of guppy, Poecilia reticulata, for the production of polyclonal antibodies[J]. Aquaculture, 2009,295(1-2): 134-137.

Page 16/28 
39. CHEN X, FANG S, WEI L, et al. Systematic evaluation of the gut microbiome of swamp eel (Monopterus albus) by 16S rRNA gene sequencing[J]. PeerJ, 2019,7: e8176.

40. KNUDSEN D, JUTFELT F, SUNDH H, et al. Dietary soya saponins increase gut permeability and play a key role in the onset of soyabean-induced enteritis in Atlantic salmon (Salmo salar L.)[J]. British Journal of Nutrition, 2008,100(1): 120-129.

41. ULLUWISHEWA D, ANDERSON R C, MCNABB W C, et al. Regulation of Tight Junction Permeability bylntestinal Bacteria and Dietary Components[J]. The Journal of Nutrition, 2011,141(5): 769-776.

42. LUISSINT A, PARKOS C A, NUSRAT A. Inflammation and the Intestinal Barrier: Leukocyte-Epithelial Cell Interactions, Cell Junction Remodeling, and Mucosal Repair[J]. Gastroenterology, 2016,151(4): 616-632.

43. ZIMMERMANN H W, SEIDLER S, GASSLER N, et al. Interleukin-8 is activated in patients with chronic liver diseases and associated with hepatic macrophage accumulation in human liver fibrosis[J]. PLoS One, 2011,6(6): e21381.

44. SUCKER A, ZHAO F, PIEPER N, et al. Acquired IFNy resistance impairs anti-tumor immunity and gives rise to T-cell-resistant melanoma lesions[J]. Nature Communications, 2017,8(1).

45. KELLY A, HOUSTON S A, SHERWOOD E, et al. Regulation of Innate and Adaptive Immunity by TGF $\beta[M]$. United States: Elsevier, 2017: 137.

46. SECOMBES C J, WANG T, HONG S, et al. Cytokines and innate immunity of fish[J]. Developmental \& Comparative Immunology, 2001,25(8-9): 713-723.

47. SAVAN R, SAKAI M. Genomics of fish cytokines[J]. Comparative Biochemistry and Physiology Part D: Genomics and Proteomics, 2006,1(1): 89-101.

48. MIN H K, KIM S, BAEK S, et al. Anthocyanin Extracted from Black Soybean Seed Coats Prevents Autoimmune Arthritis by Suppressing the Development of Th17 Cells and Synthesis of Proinflammatory Cytokines by Such Cells, via Inhibition of NF-KB[J]. PLOS ONE, 2015,10(11): e138201.

49. MCKENNA S, WRIGHT C J. Inhibiting IkappaBbeta/NFkappaB signaling attenuates the expression of select pro-inflammatory genes[J]. Journal of Cell Science, 2015,128(11): 2143-2155.

50. KANG J, SUNG M, KAWADA T, et al. Soybean saponins suppress the release of proinflammatory mediators by LPS-stimulated peritoneal macrophages[J]. Cancer Letters, 2005,230(2): 219-227.

51. RING $\varnothing$ E, ZHOU Z, VECINO J L G, et al. Effect of dietary components on the gut microbiota of aquatic animals. A never-ending story?[J]. Aquaculture Nutrition, 2016,22(2): 219-282.

52. PENG M, LUO H, KUMAR V, et al. Dysbiosis of intestinal microbiota induced by dietary oxidized fish oil and recovery of diet-induced dysbiosis via taurine supplementation in rice field eel (Monopterus albus)[J]. Aquaculture, 2019,512(2019): 734288.

53. CHUNG H, PAMP S N J, HILL J A, et al. Gut Immune Maturation Depends on Colonization with a Host-Specific Microbiota[J]. Cell, 2012,149(7): 1578-1593.

\section{Figures}




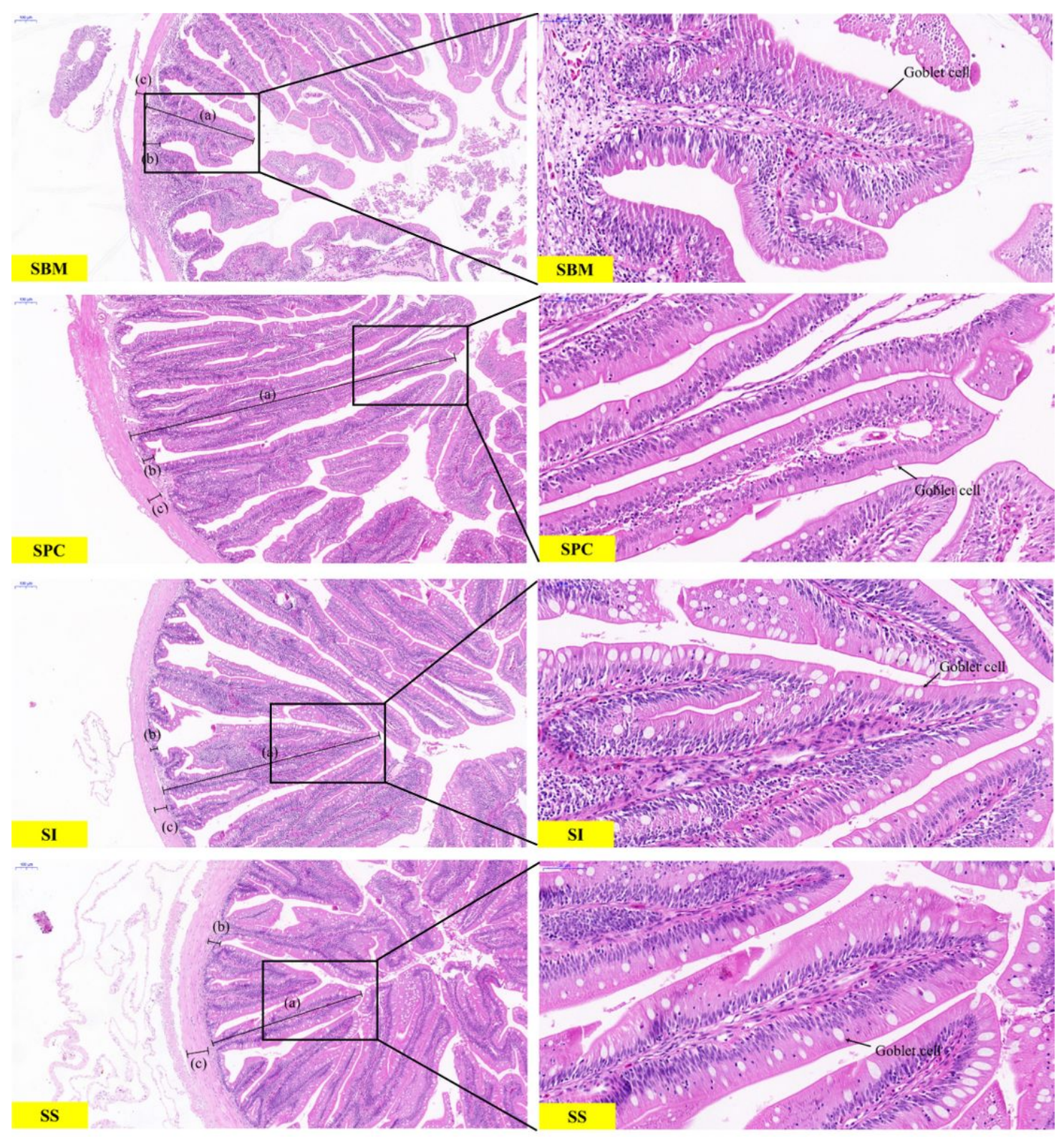

Figure 1

Effects of dietary soy isoflavone and soy saponin on the intestinal sections stained with H \& E (100 times and 400 times) of M. Albus after 8 weeks $(n=3)$. Intestinal villus height, crypt depth and intestinal muscular thickness were expressed by $(a)$, (b) and (c) in each picture, respectively. 


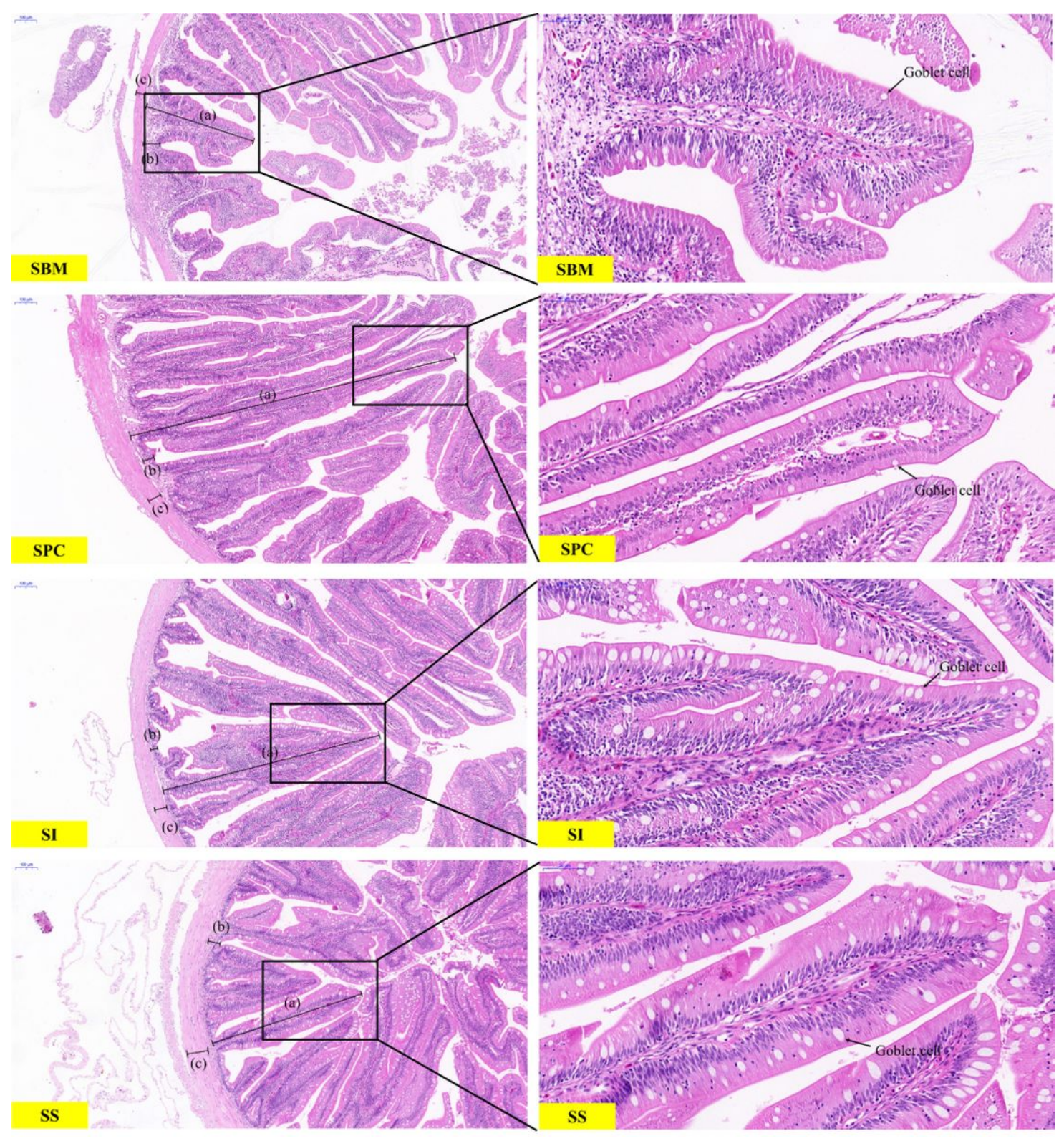

Figure 1

Effects of dietary soy isoflavone and soy saponin on the intestinal sections stained with H \& E (100 times and 400 times) of M. Albus after 8 weeks $(n=3)$. Intestinal villus height, crypt depth and intestinal muscular thickness were expressed by $(a)$, (b) and (c) in each picture, respectively. 


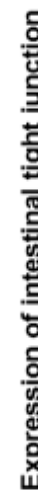
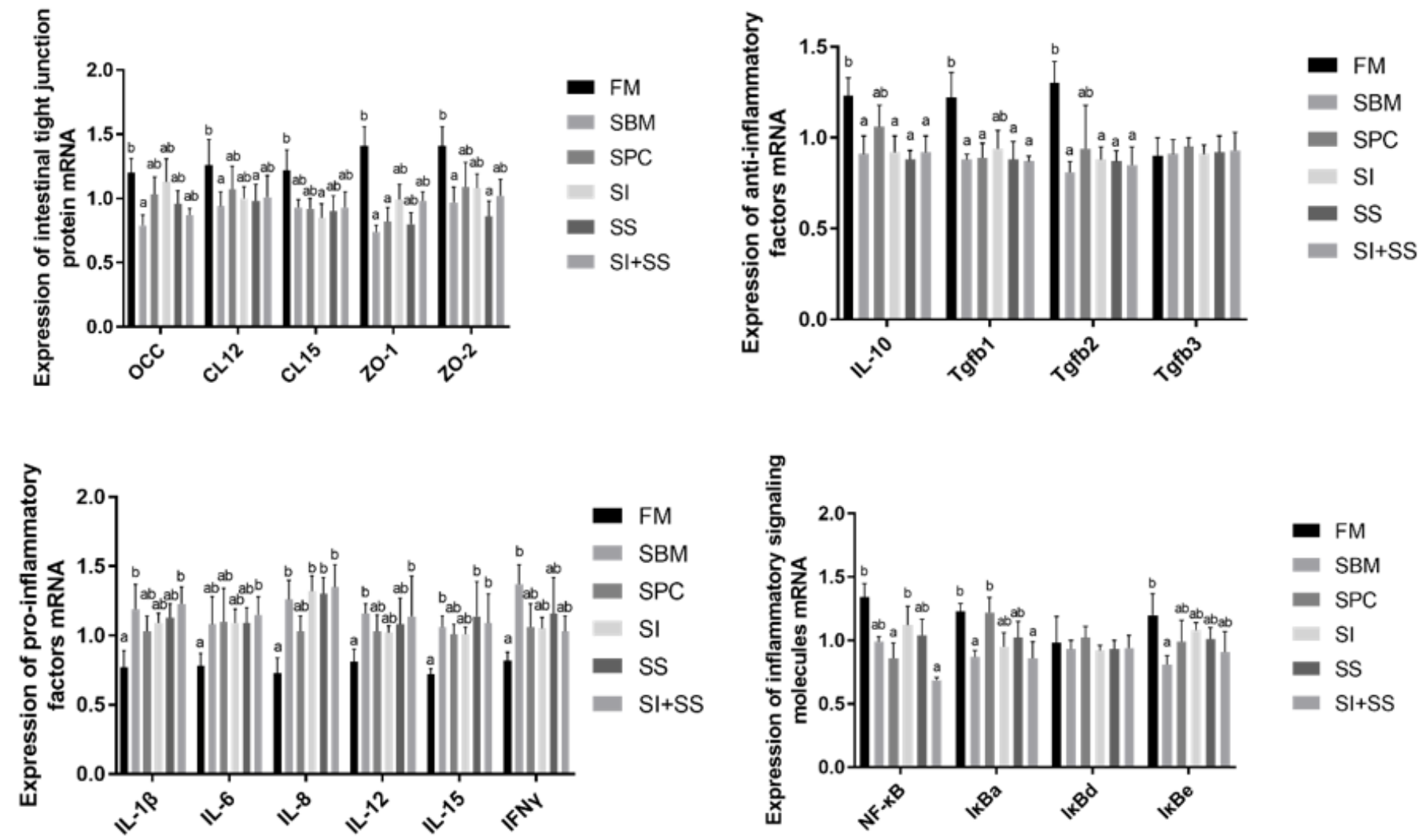

Figure 2

Effects of dietary soy isoflavone and soy saponin on intestinal factors mRNA expression of M. Albus after 8 weeks $(n=3)$. Values in each item with the same superscript or absence of superscripts are not significant different $(P>0.05)$.
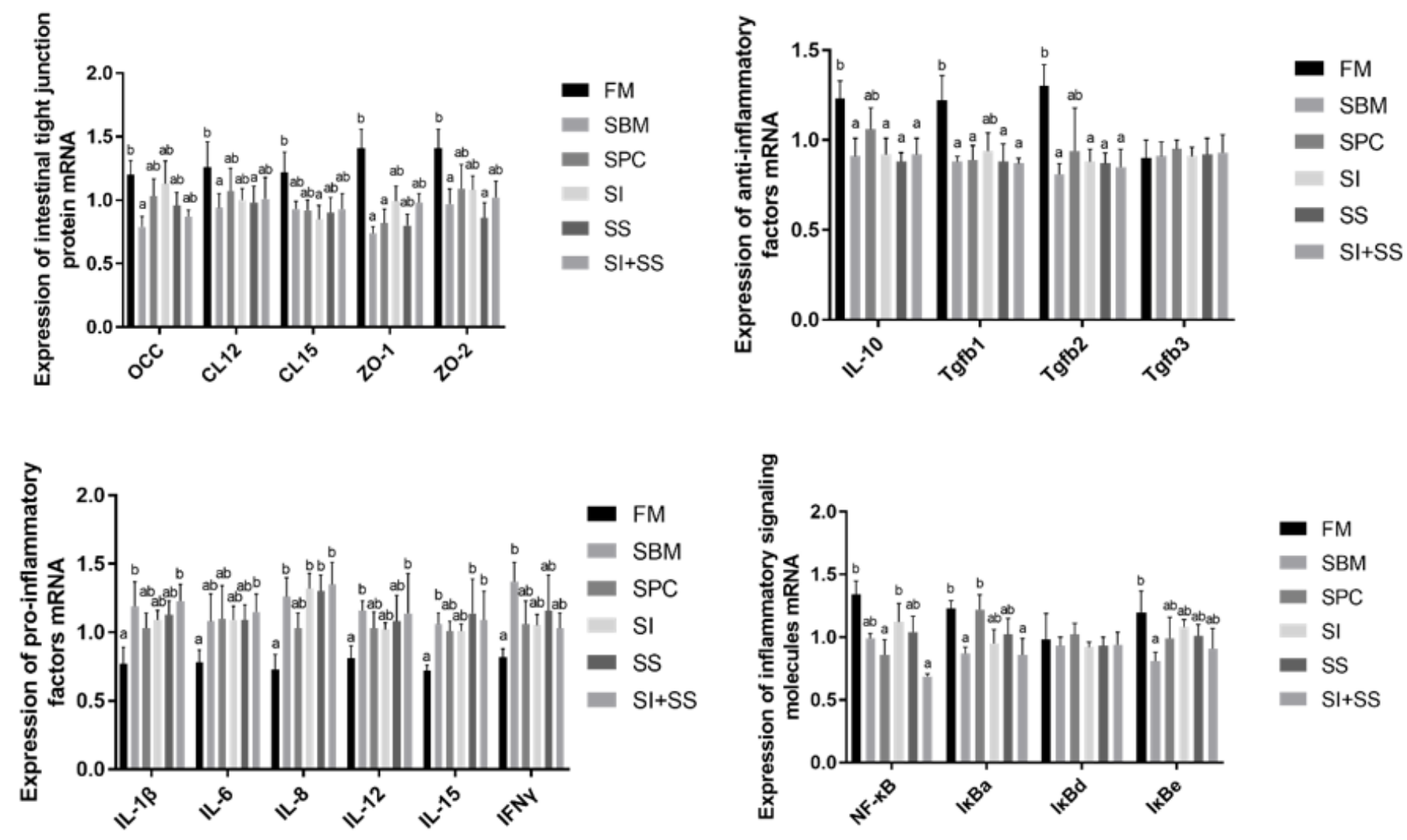

Figure 2 
Effects of dietary soy isoflavone and soy saponin on intestinal factors mRNA expression of M. Albus after 8 weeks $(n=3)$. Values in each item with the same superscript or absence of superscripts are not significant different $(P>0.05)$.
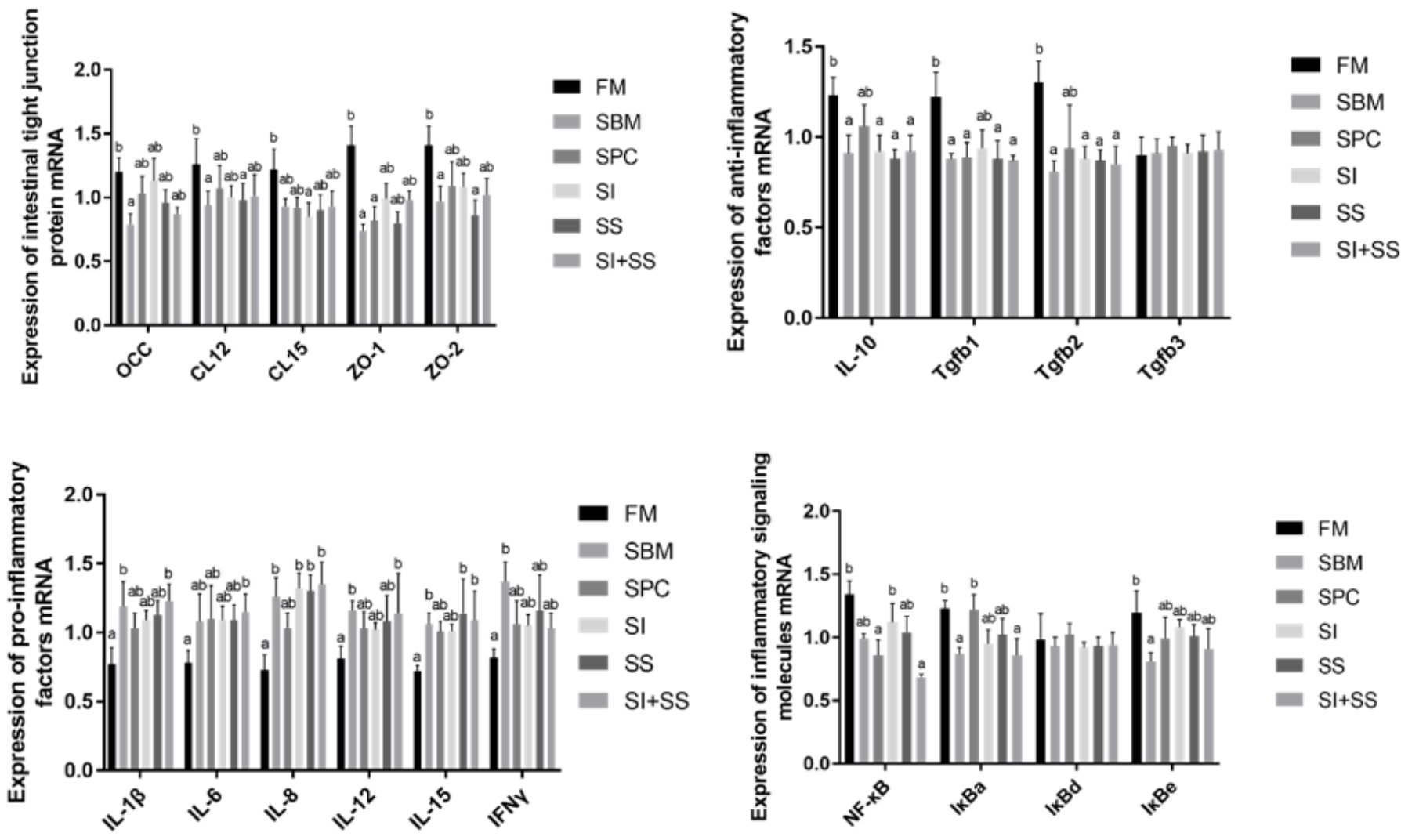

Figure 2

Effects of dietary soy isoflavone and soy saponin on intestinal factors mRNA expression of M. Albus after 8 weeks $(n=3)$. Values in each item with the same superscript or absence of superscripts are not significant different $(P>0.05)$.

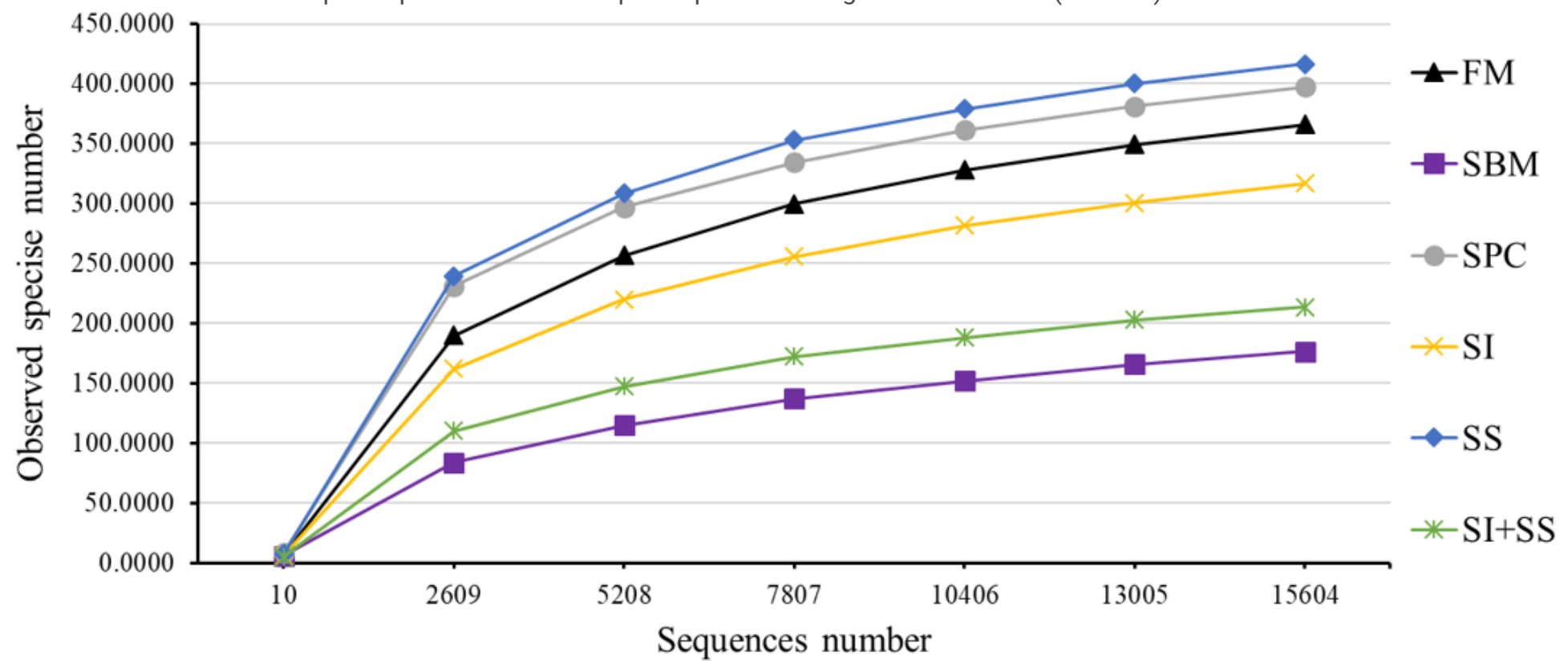

Figure 3

Effects of dietary soy isoflavone and soy saponin on OUT number of bacterial diversity index after 8 weeks $(n=3)$. 

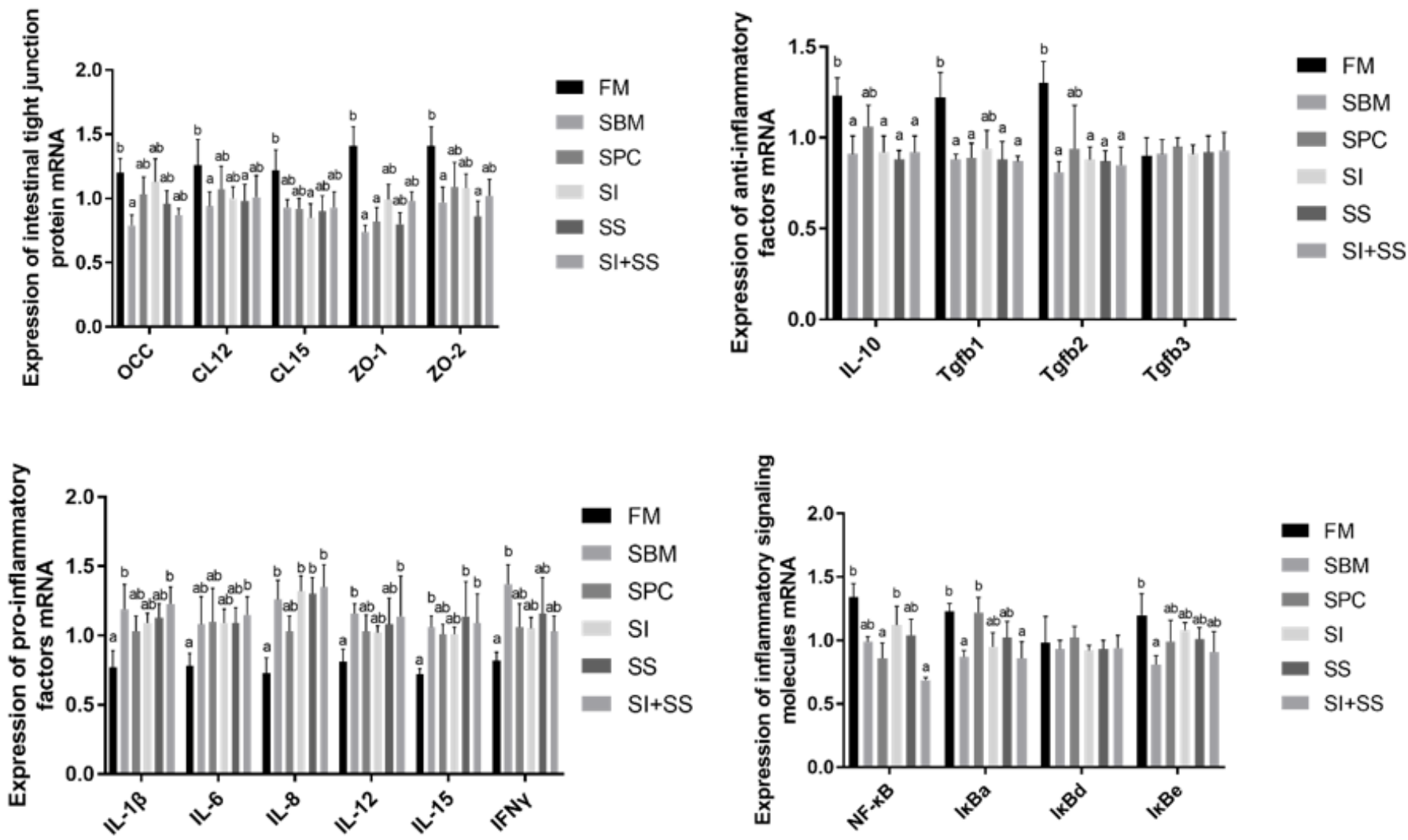

Figure 3

Effects of dietary soy isoflavone and soy saponin on intestinal factors mRNA expression of M. Albus after 8 weeks ( $\mathrm{n}=3$ ). Values in each item with the same superscript or absence of superscripts are not significant different $(P>0.05)$.

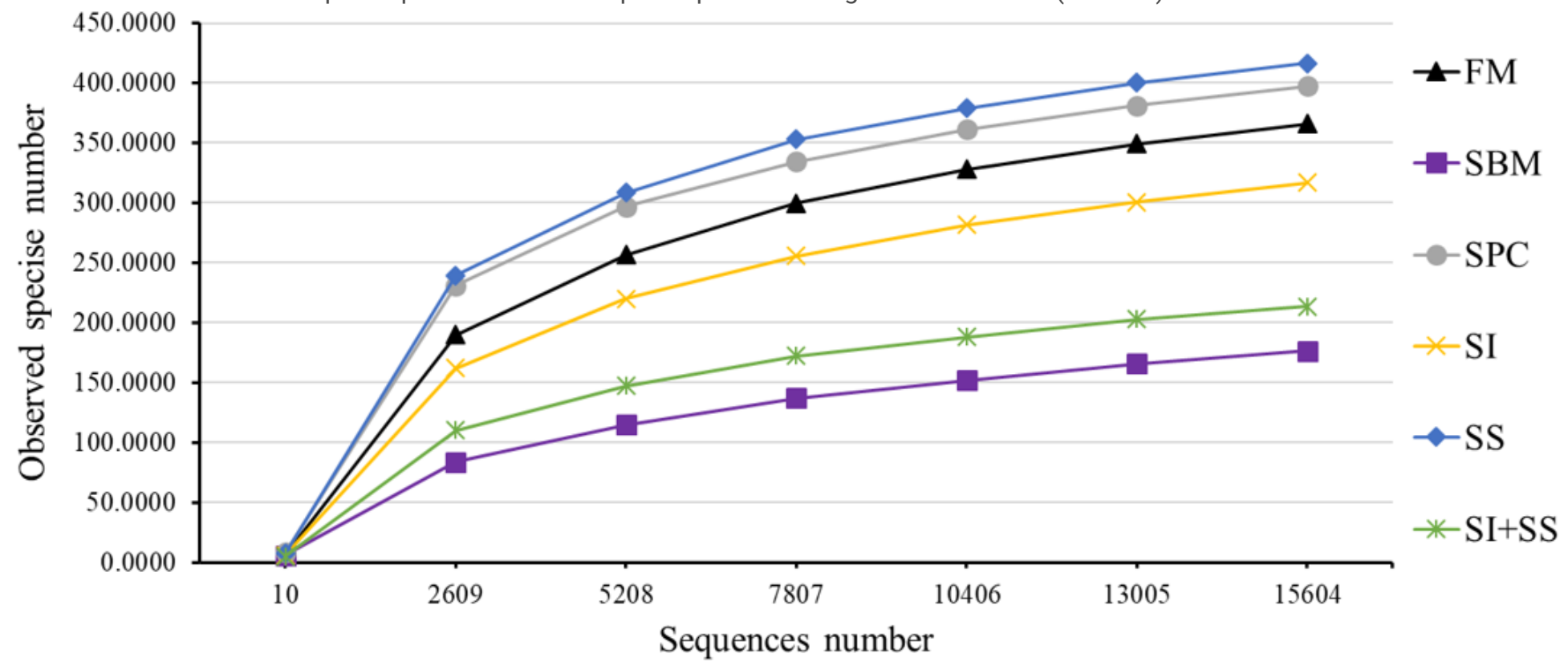

Figure 4

Effects of dietary soy isoflavone and soy saponin on OUT number of bacterial diversity index after 8 weeks ( $n=3$ ). 


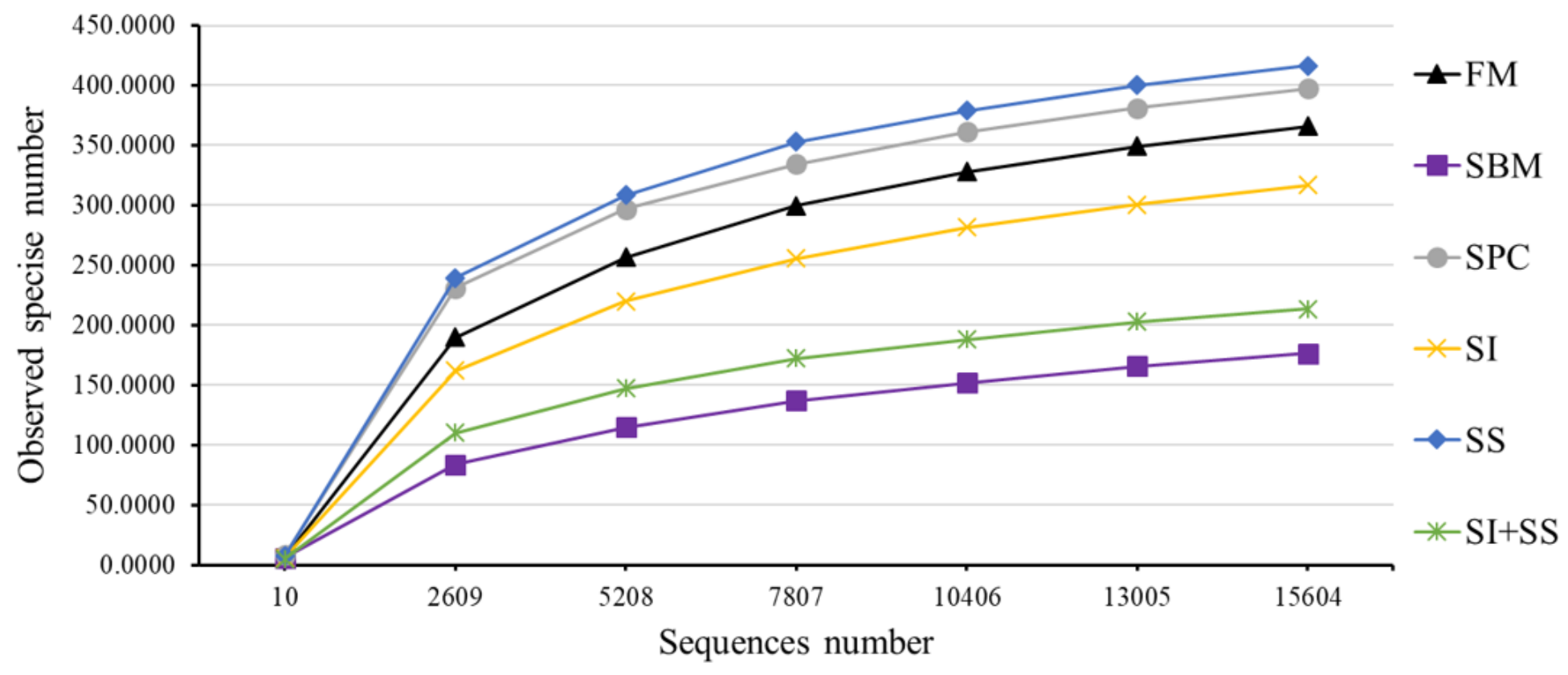

Figure 4

Effects of dietary soy isoflavone and soy saponin on OUT number of bacterial diversity index after 8 weeks ( $n=3$ ).

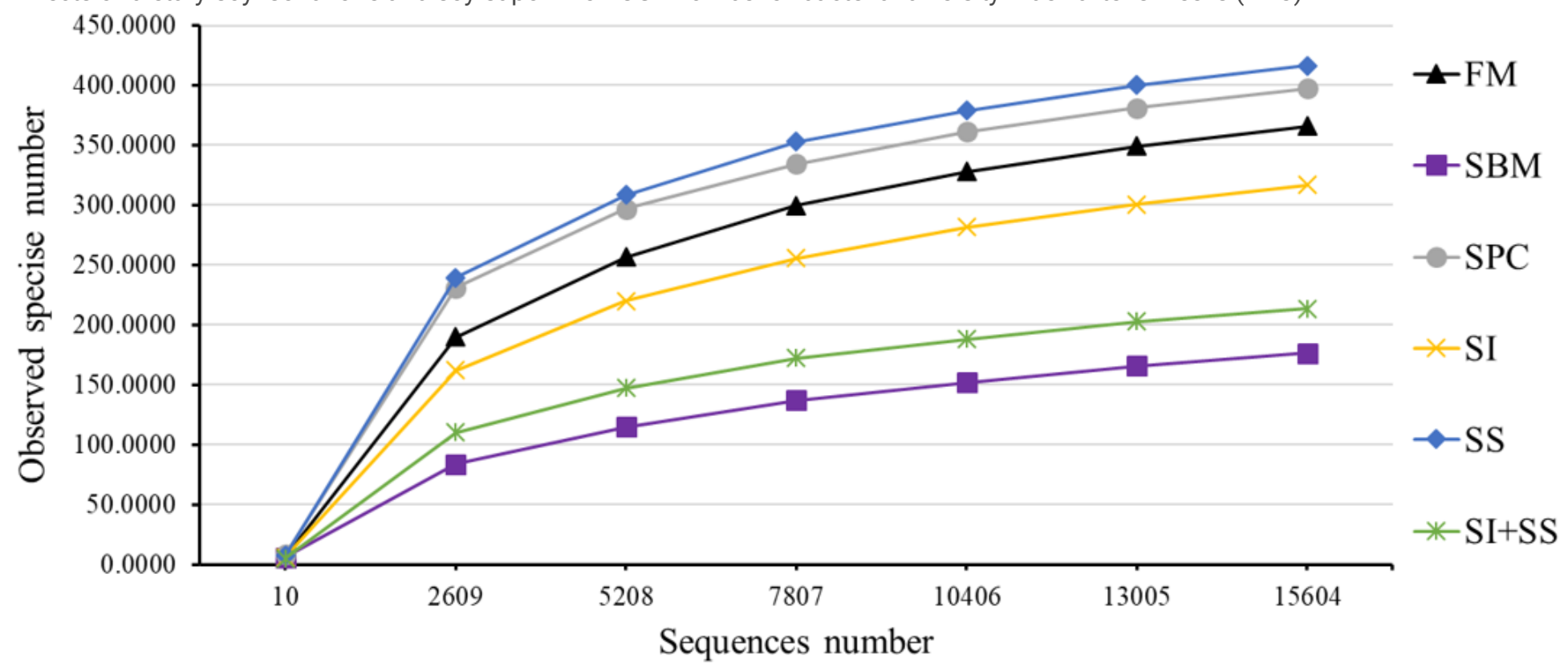

\section{Figure 4}

Effects of dietary soy isoflavone and soy saponin on OUT number of bacterial diversity index after 8 weeks $(n=3)$. 


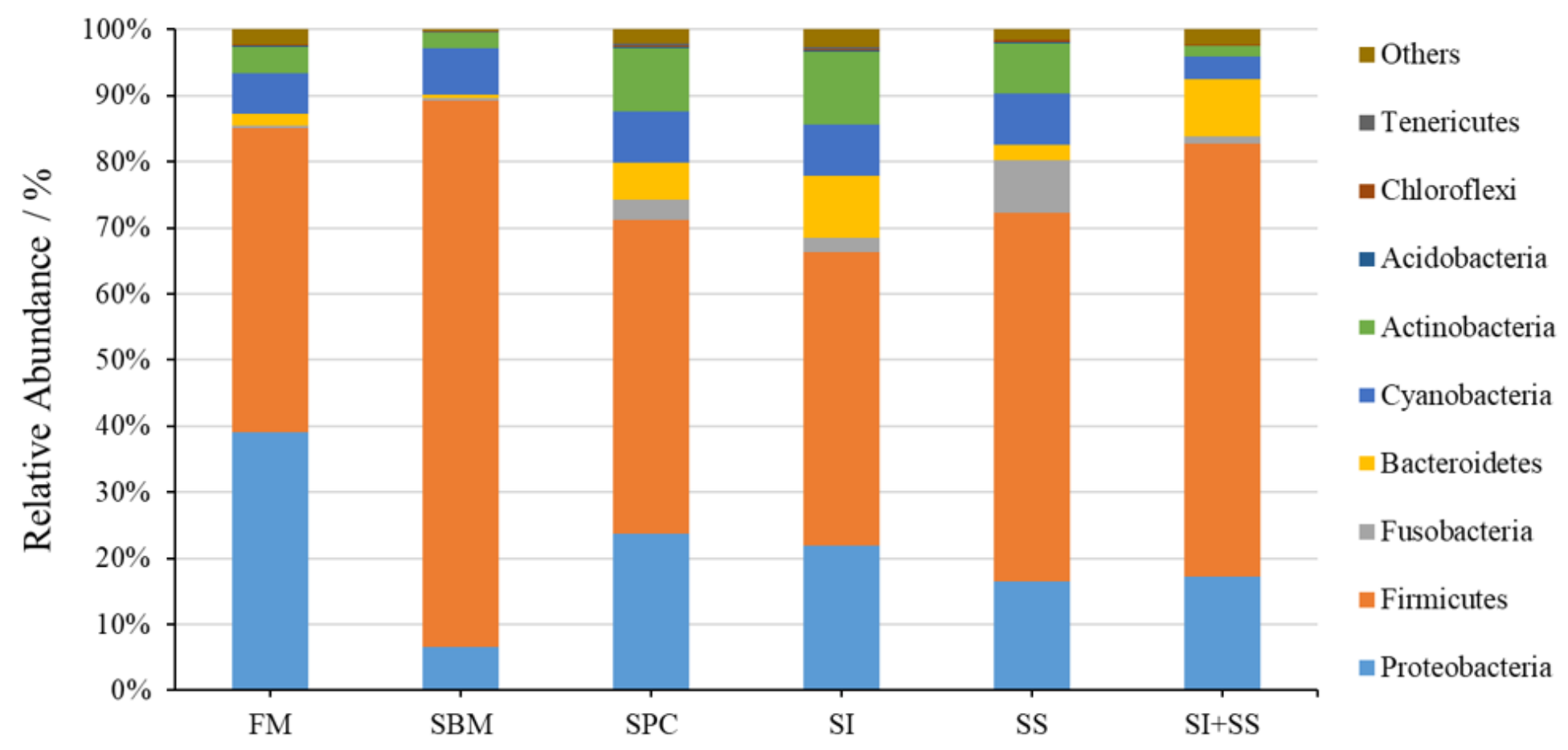

Figure 5

Effects of dietary soy isoflavone and soy saponin on phylum of bacterial diversity index after 8 weeks $(n=3)$.

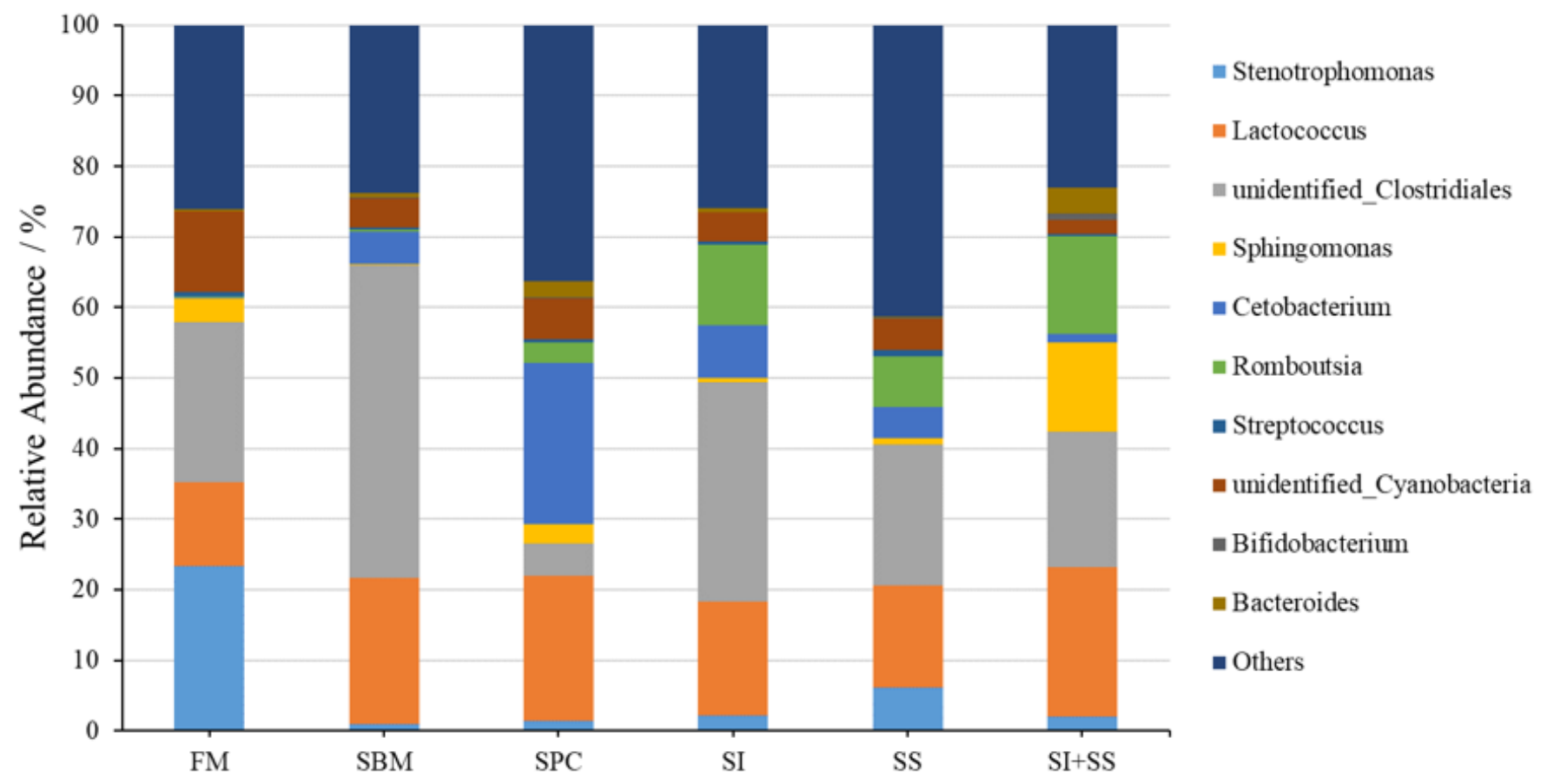

Figure 5

Effects of dietary soy isoflavone and soy saponin on genus of bacterial diversity index after 8 weeks $(n=3)$. 


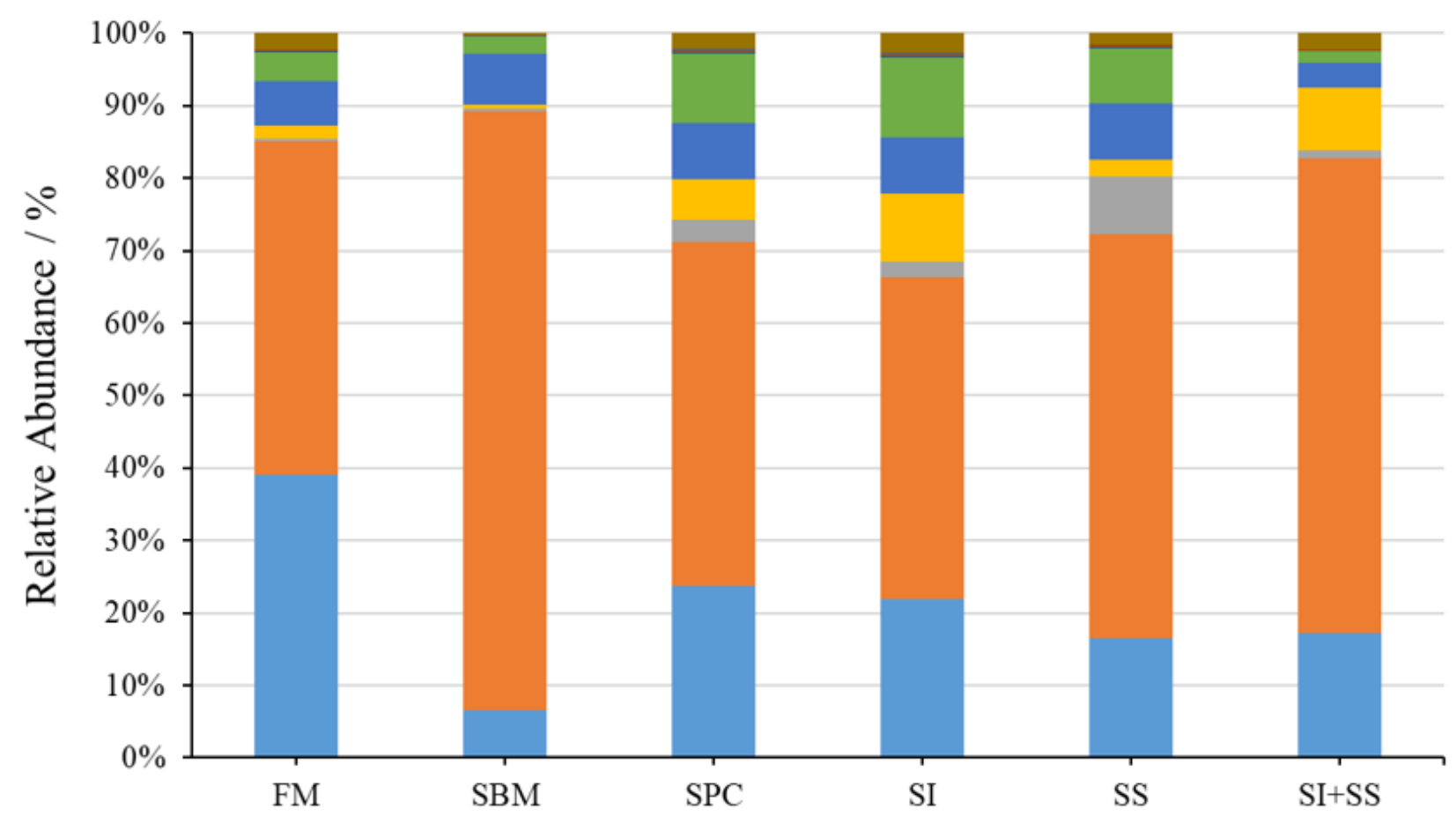

n Others

- Tenericutes

- Chloroflexi

- Acidobacteria

- Actinobacteria

= Cyanobacteria

Bacteroidetes

- Fusobacteria

airmicutes

- Proteobacteria

\section{Figure 5}

Effects of dietary soy isoflavone and soy saponin on phylum of bacterial diversity index after 8 weeks $(n=3)$.

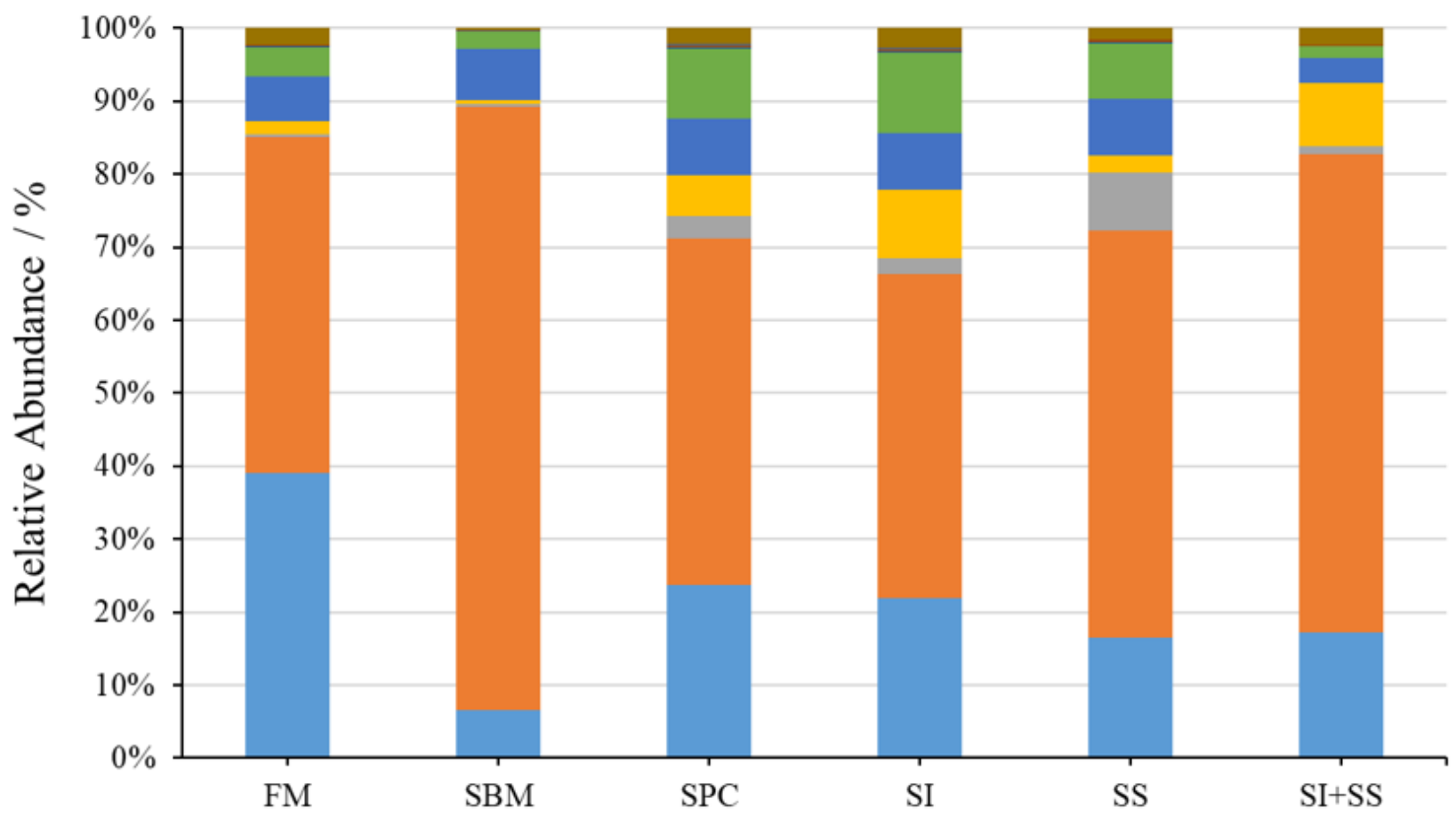

athers

- Tenericutes

- Chloroflexi

- Acidobacteria

Actinobacteria

n Cyanobacteria

Bacteroidetes

Fusobacteria

a Firmicutes

- Proteobacteria

\section{Figure 6}

Effects of dietary soy isoflavone and soy saponin on phylum of bacterial diversity index after 8 weeks $(n=3)$. 


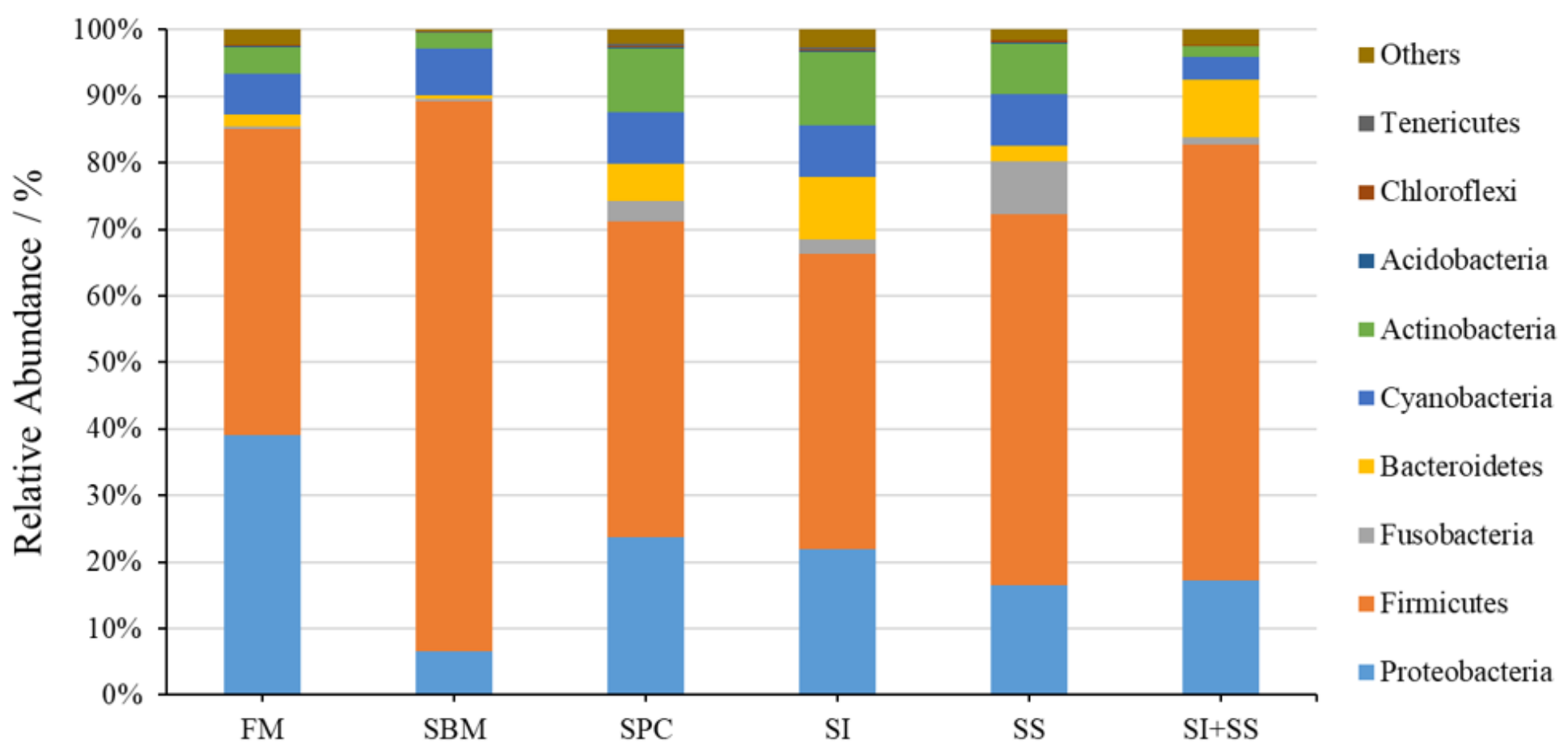

\section{Figure 6}

Effects of dietary soy isoflavone and soy saponin on phylum of bacterial diversity index after 8 weeks $(n=3)$.

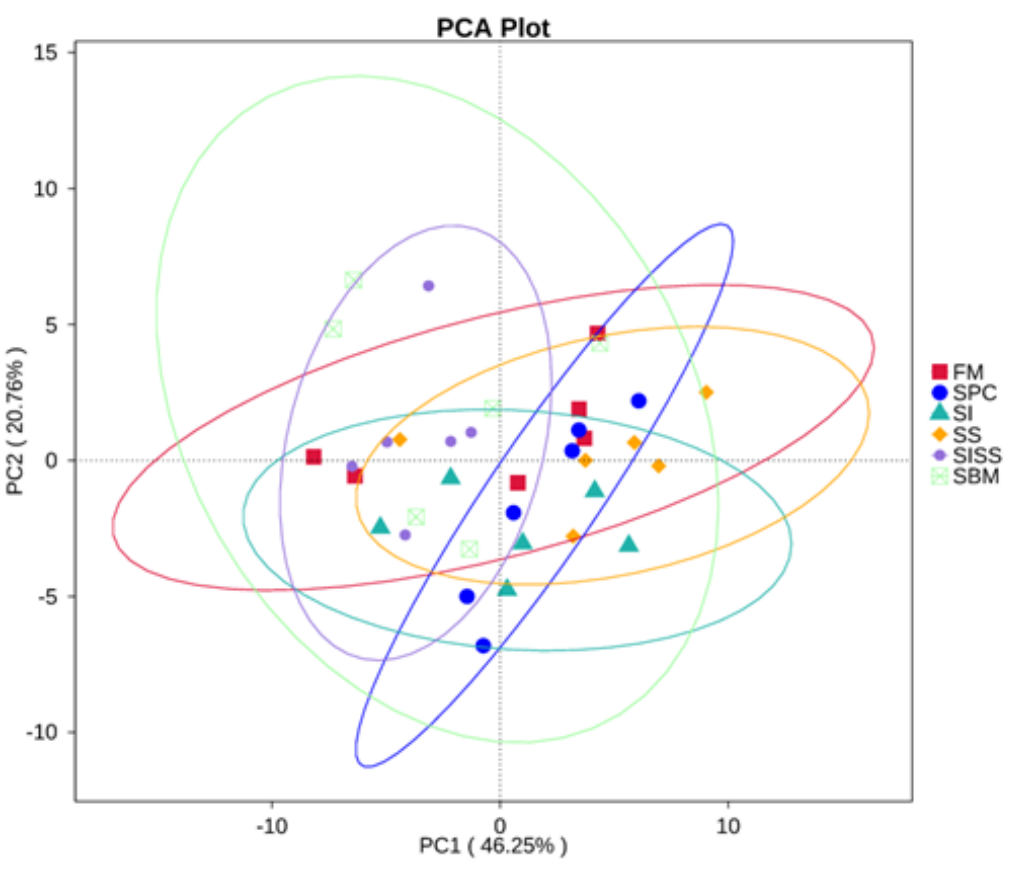

Figure 6

Effects of dietary soy isoflavone and soy saponin on gut principal component analysis (PCA) after 8 weeks $(n=3)$. Note: SISS expressed SI+SS because the analysis software can't distinguish "+". 


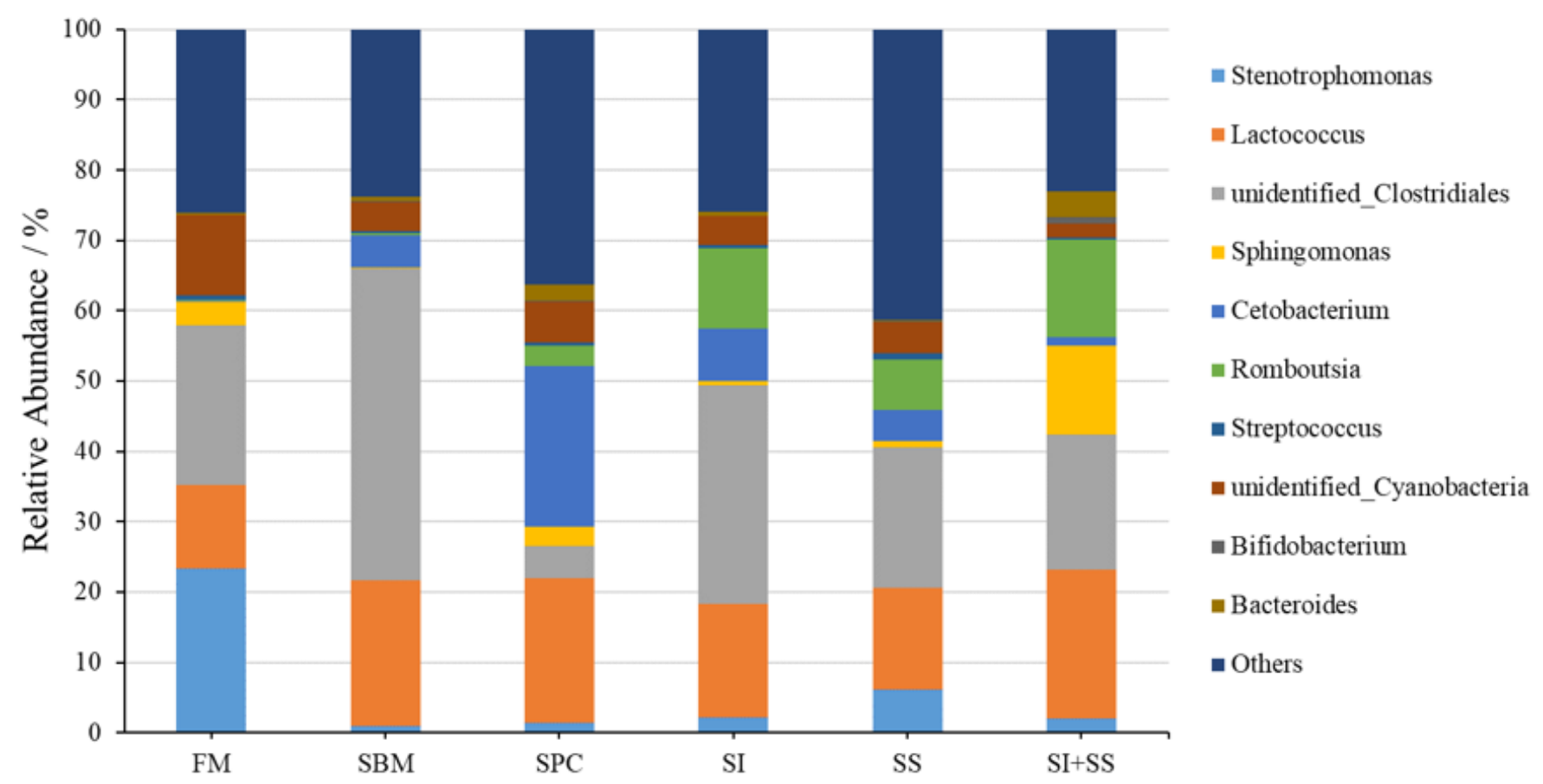

\section{Figure 7}

Effects of dietary soy isoflavone and soy saponin on genus of bacterial diversity index after 8 weeks $(n=3)$.

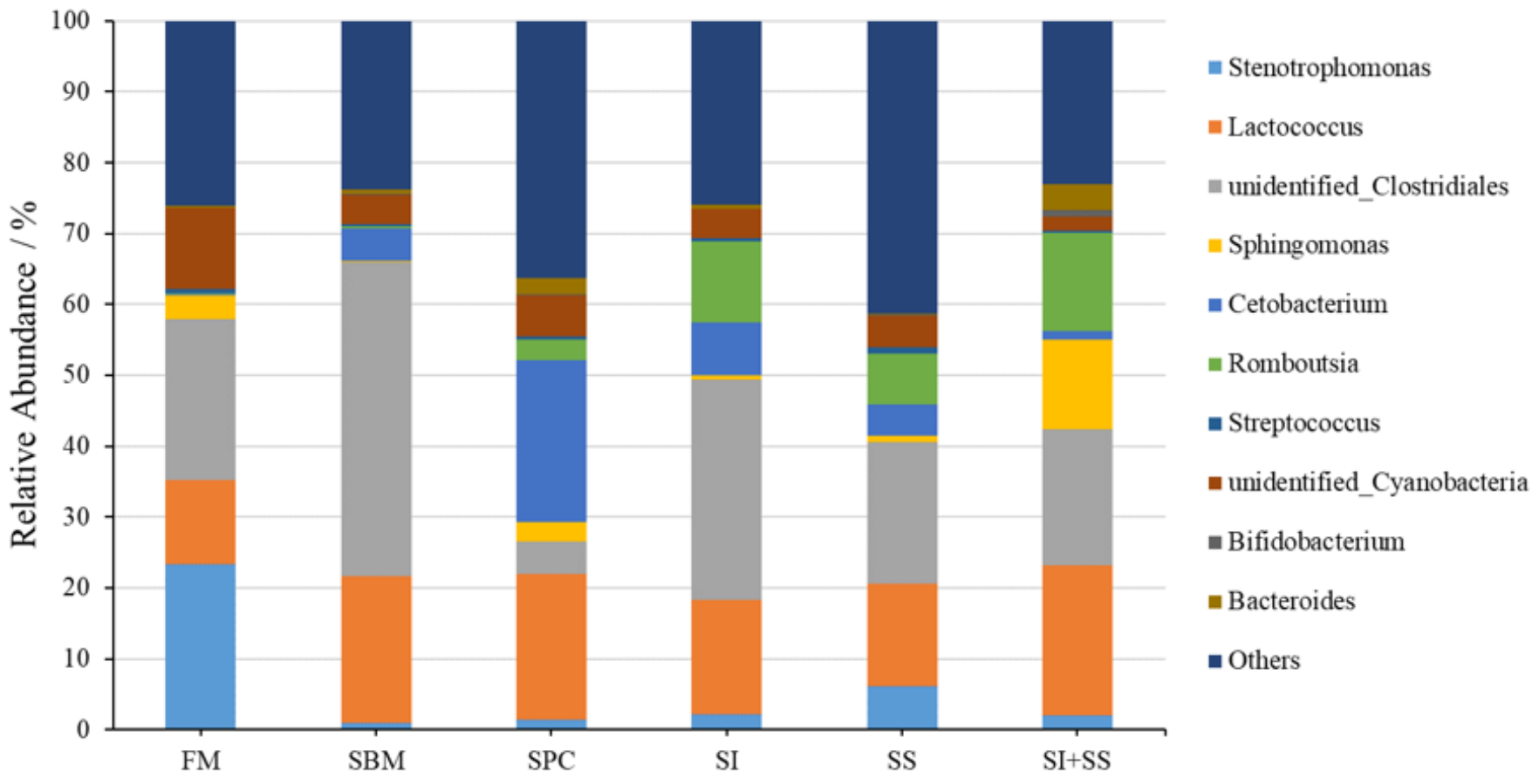

\section{Figure 8}

Effects of dietary soy isoflavone and soy saponin on genus of bacterial diversity index after 8 weeks $(n=3)$. 


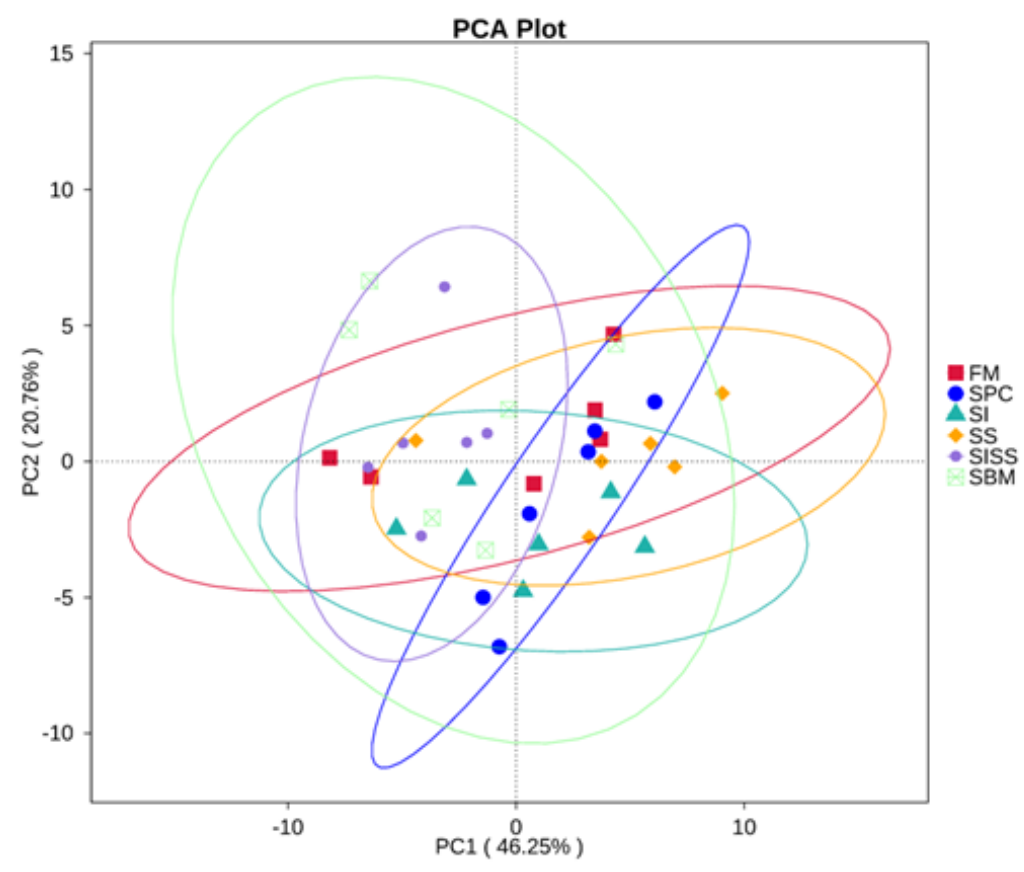

Figure 9

Effects of dietary soy isoflavone and soy saponin on gut principal component analysis (PCA) after 8 weeks $(n=3)$. Note: SISS expressed SI+SS because the analysis software can't distinguish " + ".

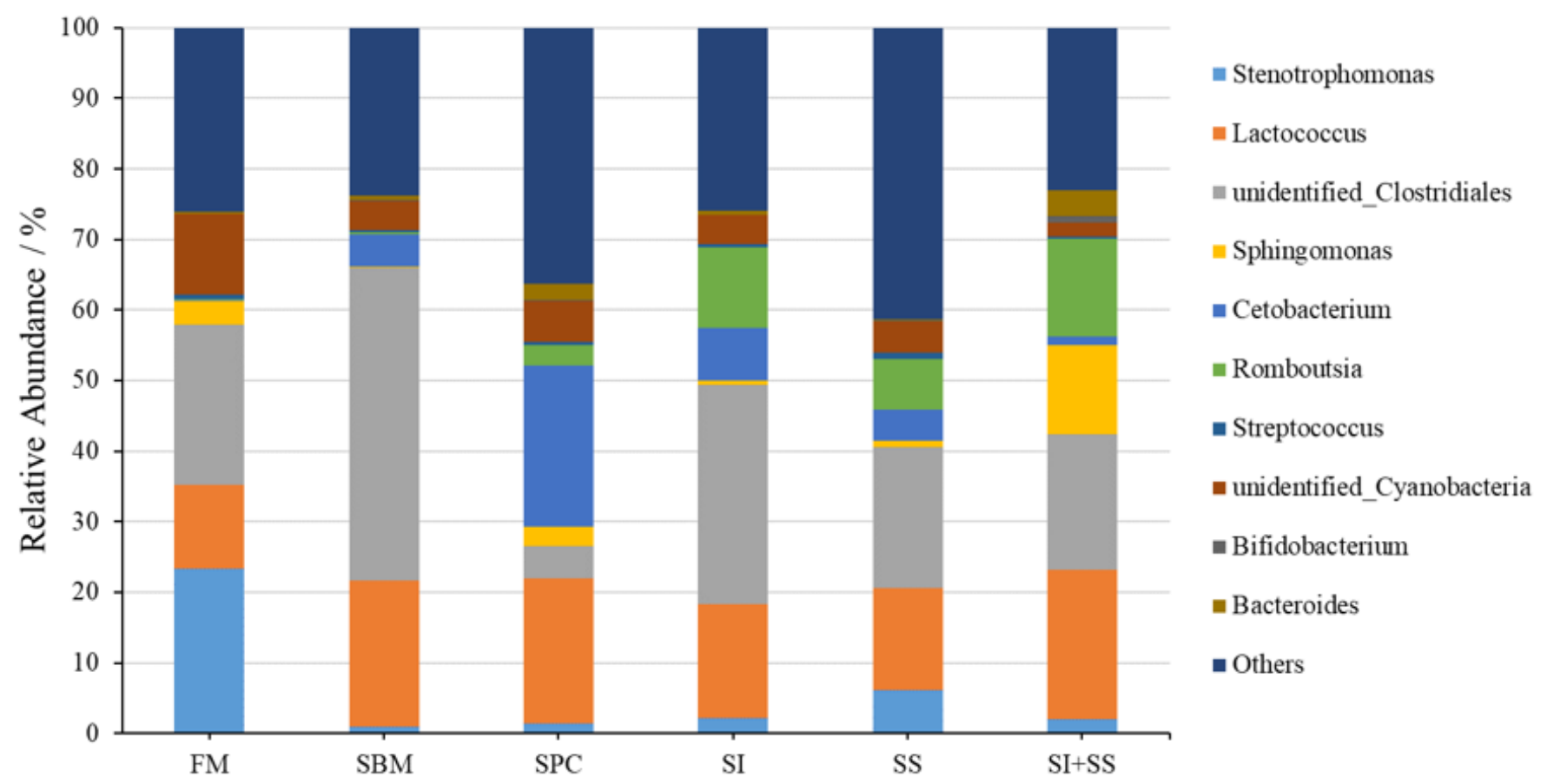

\section{Figure 9}

Effects of dietary soy isoflavone and soy saponin on genus of bacterial diversity index after 8 weeks $(n=3)$. 